\title{
A fault detection and diagnosis technique for multivariate processes using a PLS-decomposition of the measurement space
}

\author{
José L. Godoy a,b,*, Jorge R. Vega ${ }^{\text {a,c }}$, Jacinto L. Marchetti ${ }^{\text {a }}$ \\ a INTEC (CONICET and Universidad Nacional del Litoral), Güemes 3450, (3000) Santa Fe, Argentina \\ b FRP-UTN (Facultad Regional Paraná - Universidad Tecnológica Nacional), Almafuerte 1033 - (3100) Paraná, Argentina \\ c FRSF-UTN (Facultad Regional Santa Fe - Universidad Tecnológica Nacional), Lavaisse 610, (3000) Santa Fe, Argentina
}

\section{A R T I C L E I N F O}

\section{Article history:}

Received 2 November 2012

Received in revised form 5 July 2013

Accepted 12 July 2013

Available online $\mathrm{xxxx}$

\section{Keywords:}

PLS

Process monitoring

Fault detection

Fault diagnosis

Fault isolation

Contribution analysis

\begin{abstract}
A B S T R A C T
A new statistical monitoring technique based on partial least squares (PLS) is proposed for fault detection and di- 24 agnosis in multivariate processes that exhibit collinear measurements. A typical PLS regression (PLSR) modeling 25 strategy is first extended by adding the projections of the model outputs to the latent space. Then, a PLS- 26 decomposition of the measurements into four terms that belongs to four different subspaces is derived. In $27 \mathbf{Q 2}$ order to online monitor the PLS-projections in each subspace, new specific statistics with non-overlapped do- 28 mains are combined into a single index able to detect process anomalies. To reach a complete diagnosis, a further 29 decomposition of each statistic was defined as a sum of variable contributions. By adequately processing all this 30 information, the technique is able to: i) detect an anomaly through a single combined index, ii) diagnose the 31 anomaly class from the observed pattern of the four component statistics with respect to their respective confi- 32 dence intervals, and iii) identify the disturbed variables based on the analysis of the main variable contributions 33 to each of the four subspaces. The effectiveness observed in the simulated examples suggests the potential appli- 34 cation of this technique to real production systems.
\end{abstract}

\section{Introduction}

Statistical process monitoring (SPM) applies multivariate statistics and machine learning methods to product quality and production control, fault detection and diagnosis, and estimation of fault/fault-free magnitudes. The SPM approaches based on historical operating data are useful when applied to process having a large number of measured variables and when causal models are unavailable [1]. In particular, latent variable (LV) models are more adequate than causal models to deal with monitoring tasks. Along the last two decades, several strategies based on LV models have been proposed for multivariate SPM [2-7]. When a process operates under normal conditions (i.e., when only 'common-cause' variations are present), then the correlation structure underlying the measured data can be adequately described through LV models. Monitoring techniques based on LV are useful for detecting and diagnosing abnormal behaviors in complex multivariate processes; and therefore they are of great interest for their use in industrial applications [8]. For instance, partial least squares (PLS) is often applied in Process Analytical Technology (PAT) projects, where most applications have successfully been used for monitoring biotechnological processes of the pharmaceutical industry [9].

\footnotetext{
* Corresponding author at: INTEC (CONICET and Universidad Nacional del Litoral), Güemes 3450, (3000) Santa Fe, Argentina. Tel.: + 54342 4559175; fax: + 54342 4550944.

E-mail address: jlgodoy@santafe-conicet.gov.ar (J.L. Godoy).
}

Several fault detection indexes based on LV models can be used to 61 alert on the presence of possible anomalies during the process opera- 62 tion. An alarm signal typically appears when an index exceeds its 63 predefined control limit. Once a fault is detected, the fault diagnosis is 64 made by analyzing the contributions of each measured variable to the 65 specific index that caused the alarm. An essential requirement for fault 66 diagnosis is to avoid misdiagnosis. For example, in the case of a fault 67 in a sensor or in an actuator, only one variable associated to the faulty 68 device should ideally contribute to the index with a high positive 69 value. Contribution charts basically calculate the contributions of the 70 variables in a fault situation and select the variables with large contribu- 71 tions as indicators of the probable cause of the fault [10]. Thus, an anal- 72 ysis of well-defined contributions should have the following desirable 73 properties: i) in absence of faults, all contributions would exhibit similar 74 low average values, thus determining a normal baseline level; ii) in the 75 presence of a single fault (i.e., a fault that is due to only one variable), the 76 contribution corresponding to the faulty variable should be large, and 77 iii) in the presence of a multiple fault (e.g., multiple sensor failures), 78 the abnormally large contribution of each faulty variable must be great- 79 er than the rest [11].

In medium and large scale processes (such as chemical manufactur- 81 ing plants, food industries, etc.), there are a large number of controlled, 82 manipulated, and process variables. When a multivariate process 83 operates under normal conditions, then the measurement space 84 spanned by the measured variables exhibits a non-full or deficient 85 rank. In such a case, a model of the correlation structure among all 86 
measured variables is required for reaching a proper process monitoring. Methods of projection to LVs can be used to transform noisy collinear data into well-conditioned and reduced-dimension data that preserve all the useful information about the process, thus allowing the capture of the 'common-cause' subspace [7]. These data-driven methods typically use historic data (collected during normal operating conditions) to develop a LV model able to effectively explain the 'common-cause' variability. Several of these monitoring techniques (such as PCA: principal component analysis, ICA: independent component analysis, Kernel PCA, and Kernel ICA) treat the data without differentiating outputs from inputs [12-15]. In contrast, a PLS regression (PLSR) model is closer to the intrinsic structure of multi-input multi-output process [16,17], because it allows the elimination of some undesired input variables from the original pre-sets (e.g., those interfering the regression model) [18,19].

In the last years, several tools based on PLSR have been proposed for monitoring industrial processes [18,20-23]. Simultaneously, some efforts have also been aimed at improving the fundamentals of PLSR $[24,25]$. The development of surveillance PLSR tools able to detect anomalies or poor process performances is undoubtedly an active field of current research.

This article aims at developing an online data processing technique useful for helping process engineers to reach a swift identification of the origin of a fault when the monitoring system trigger an alarm signal. The paper is organized as follows. Section 2 describes the fundamentals of an extended PLSR modeling and their geometric properties, which was needed in order to obtain a new online PLS-decomposition of the measurements with associated statistics and contribution analysis. Section 3 presents the main statistical monitoring tools and describes their roles in the measurement PLS-decomposition. Section 4 discusses some simulation tests based on both static and dynamic systems. Finally, the main conclusions are presented in Section 5.

\section{Extended PLSR modeling}

The PLSR model developed here is calculated by simultaneously deflating the data matrices with the classical PLS-NIPALS algorithm [26]. This procedure gives better results for multivariate prediction and process monitoring than other alternative PLS algorithms [25]. Besides, the simultaneous deflation on both data matrices allows the detection of predictor variables playing an interfering effect [18].

Consider a process with $m$ measured input variables plus $p$ measured output variables. Assume that $N$ measurements of each variable are collected while the process is operating under normal conditions. In order to build a model, the $N$ multivariate measurements are arranged into a predictor matrix $\mathbf{X}=\left[\mathbf{x}_{1} \ldots \mathbf{x}_{N}\right]^{\prime}(N \times m)$ consisting of $N$ samples of $m$

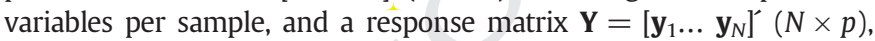
with $N$ samples of $p$ variables per sample. Then, PLSR can be used to find a regression model between the measurement vectors $\mathbf{x}=$ $\left[x_{1} \ldots x_{m}\right]^{\prime}$ and $\mathbf{y}=\left[y_{1} \ldots y_{p}\right]^{\prime}$, even when their correlation matrices $\left(\mathbf{R}_{\mathbf{x}}\right.$ and $\left.\mathbf{R}_{\mathbf{y}}\right)$ are both positive semi-definite (i.e., $\mathbf{X}$ and $\mathbf{Y}$ have collinear variables). The method produces a projection of $\mathbf{X}$ and $\mathbf{Y}$ into lowdimension spaces defined by the number $A$ of $L V$ which are then regressed. At each iteration $a$, the implicit objective of the PLSNIPALS algorithm (see Table 1) is to find a solution to the following optimization problem [25]:

$\max _{\mathbf{w}_{a}, \mathbf{q}_{a}}\left(\mathbf{w}_{a}^{\prime} \mathbf{X}_{a}^{\prime} \mathbf{Y}_{a} \mathbf{q}_{a}\right) \quad$ subject to : $\left\|\mathbf{w}_{a}\right\|=1,\left\|\mathbf{q}_{a}\right\|=1$.

In Eq. (1), $\mathbf{X}_{a}$ and $\mathbf{Y}_{a}$ stand for the $a$-times deflated versions of $\mathbf{X}_{1}=$ $\mathbf{X}$ and $\mathbf{Y}_{1}=\mathbf{Y}$ respectively. The number $A$ of deflations to be made is determined by checking the residual matrices until the leftover information can no longer be modeled [18]. In this way, the PLSR modeling strategy produces an external and an internal model. The external
Table 1

(X, Y)-deflated PLS-NIPALS algorithm. Outputs: P, Q B, T, and U.

Center the columns of $\mathbf{X}, \mathbf{Y}$ to zero mean and scale them to unit variance.

Set $a=1, \mathbf{X}_{1}=\mathbf{X}, \mathbf{Y}_{1}=\mathbf{Y}$. (Initialization)

1. Set $\mathbf{t}_{a}^{0}$ equal to the maximum-variance column of $\mathbf{X}_{a}$

Set $\mathbf{u}_{a}$ equal to the maximum-variance column of $\mathbf{Y}_{a}$.

2. $\mathbf{w}_{a}=\mathbf{X}_{a}^{\prime} \mathbf{u}_{a} /\left\|\mathbf{X}_{a}^{\prime} \mathbf{u}_{a}\right\|,\left(\left\|\mathbf{w}_{a}\right\|=1\right)$.

3. $\mathbf{t}_{a}=\mathbf{X}_{a}^{\prime} \mathbf{w}_{a}$.

4. $\mathbf{q}_{a}=\mathbf{Y}_{a}^{\prime} \mathbf{t}_{a} /\left\|\mathbf{Y}_{a}^{\prime} \mathbf{t}_{a}\right\|,\left(\left\|\mathbf{q}_{a}\right\|=1\right)$.

5. $\mathbf{u}_{a}=\mathbf{Y}_{a}^{\prime} \mathbf{q}_{a}$.

If $\left\|\mathbf{t}_{a}^{0}-\mathbf{t}_{a}\right\|<\varepsilon$, go to step 6, else set $\mathbf{t}_{a}^{0}=\mathbf{t}_{a}$ and return to step 2 .

6. $\mathbf{p}_{a}^{*}=\mathbf{X}_{a}^{\prime} \mathbf{t}_{a} /\left(\mathbf{t}_{a}^{\prime} \mathbf{t}_{a}\right), \mathbf{p}_{a}=\mathbf{p}_{a}^{*} /\left\|\mathbf{p}_{a}^{*}\right\|,\left(\left\|\mathbf{p}_{a}\right\|=1\right) .{ }^{\#}$

7. $\mathbf{t}_{a}=\mathbf{t}_{a}\left\|\mathbf{p}_{a}^{*}\right\|, \mathbf{w}_{a}=\mathbf{w}_{a}\left\|\mathbf{p}_{a}^{*}\right\|,\left(\left\|\mathbf{w}_{a}\right\| \neq 1\right)$.

8. $b_{a}=\mathbf{u}_{a}^{\prime} \mathbf{t}_{a} /\left(\mathbf{t}_{a}^{\prime} \mathbf{t}_{a}\right)$, (inner regression).

9. $\mathbf{X}_{a+1}=\mathbf{X}_{a}-\mathbf{t}_{a} \mathbf{p}_{a}^{\prime}, \mathbf{Y}_{a+1}=\mathbf{Y}_{a}-b_{a} \mathbf{t}_{a} \mathbf{q}_{a}^{\prime}$, (deflations).

10. $\mathbf{P} \leftarrow \mathbf{p}_{a}, \mathbf{Q} \leftarrow \mathbf{q}_{a}, \mathbf{B} \leftarrow b_{a}, \mathbf{T} \leftarrow \mathbf{t}_{a}, \mathbf{U} \leftarrow \mathbf{u}_{a}$, (save data to matrices).

Set $a=a+1$. If $a>A$, then stop; else return to step 1 .

\# : Compensation of scales by previous normalization.

model decomposes $\mathbf{X}$ and $\mathbf{Y}$ into score vectors ( $\mathbf{t}_{a}$ and $\mathbf{u}_{a}$ ), loading vec- 149 tors $\left(\mathbf{p}_{a}\right.$ and $\left.\mathbf{q}_{a}\right)$, and residual error matrices $\left(\widetilde{\mathbf{X}}\right.$ and $\left.\widetilde{\mathbf{Y}}_{2}\right)$, as follows:

$\mathbf{X}=\sum_{a=1}^{A} \mathbf{t}_{a} \mathbf{p}_{a}^{\prime}+\widetilde{\mathbf{X}}=\mathbf{T P}^{\prime}+\widetilde{\mathbf{X}}, \quad \mathbf{P}=\left[\mathbf{p}_{1} \ldots \mathbf{p}_{A}\right]$,

$\mathbf{Y}=\sum_{a=1}^{A} \mathbf{u}_{a} \mathbf{q}_{a}^{\prime}+\widetilde{\mathbf{Y}}_{2}=\mathbf{U} \mathbf{Q}^{\prime}+\widetilde{\mathbf{Y}}_{2}, \quad \mathbf{Q}=\left[\mathbf{q}_{1} \ldots \mathbf{q}_{A}\right]$

where $\mathbf{T}=\left[\mathbf{t}_{1} \ldots \mathbf{t}_{A}\right]$ and $\mathbf{U}=\left[\mathbf{u}_{1} \ldots \mathbf{u}_{A}\right]$ are orthogonal by columns. In the $15 \mathrm{~g}$ internal model, $\mathbf{t}_{a}$ is linearly regressed against the $y$-score vector $\mathbf{u}_{a}$, i.e., 155

$\mathbf{U}=\mathbf{T B}+\widetilde{\mathbf{U}}, \mathbf{B}=\operatorname{diag}\left(b_{1} \ldots b_{A}\right)$

where $b_{1} \ldots b_{A}$ are the regression coefficients determined by minimiza- 158 tion of the residuals $\widetilde{\mathbf{U}}$.

Call $\mathbf{R}$ and $\mathbf{S}$ the pseudo-inverses of $\mathbf{P}^{\prime}$ and $\mathbf{Q}^{\prime}$, respectively (i.e., $\mathbf{P} \mathbf{R}=\mathbf{I} 159$ and $\mathbf{Q} \mathbf{S}=\mathbf{I}$ ). Then, for new $\mathbf{X}$ and $\mathbf{Y}$, the predictions of $\mathbf{T}$ and $\mathbf{U}$ are direct- 160 ly obtained from Eqs. (2) and (3), as:

$\mathbf{T}=\mathbf{X R}, \quad \mathbf{R}=\left[\mathbf{r}_{1} \ldots \mathbf{r}_{A}\right]$,

$\mathbf{U}=\mathbf{Y S}, \quad \mathbf{S}=\left[\mathbf{S}_{1} \ldots \mathbf{S}_{A}\right]$.

Since the row space of $\widetilde{\mathbf{X}}$ belongs to the null-space of $\mathbf{R}$, then $\widetilde{\mathbf{X}} \mathbf{R}=0166$ [27]. Similarly for $\widetilde{\mathbf{Y}}_{2}$, that belongs to the null space $\mathbf{S}$, and consequently 167 $\widetilde{\mathbf{Y}}_{2} \mathbf{S}=0$. Hence, by combining Eqs. (3)-(5), the following prediction 168 model is obtained:

$\mathbf{Y}=\mathbf{X R B Q ^ { \prime }}+\widetilde{\mathbf{U}} \mathbf{Q}^{\prime}+\widetilde{\mathbf{Y}}_{2}=\widehat{\mathbf{Y}}+\widetilde{\mathbf{Y}}_{1}+\widetilde{\mathbf{Y}}_{2}$,

where $\widetilde{\mathbf{Y}}_{2}=\mathbf{Y}-\mathbf{Y S Q}^{\prime}$ and $\widetilde{\mathbf{Y}}_{1}=\mathbf{Y S Q}^{\prime}-\widehat{\mathbf{Y}}$ are the projection and transfor- 170 mation error matrices, respectively. Hence, we have extended the PLSR 172 model description by adding the projection of $\mathbf{Y}$ to $\mathbf{U}$ (Eq. (6)), which al- 173 lows the decomposition of the prediction error $\widetilde{\mathbf{Y}}$ into two terms: $\widetilde{\mathbf{Y}}_{1}$ and 174 $\widetilde{\mathbf{Y}}_{2}$ (Eq. (7)).

\subsection{PLS-decomposition of the input and output spaces}

After synthesizing an "in-control" PLSR model, the measurement 177 vectors $\mathbf{x} \in \mathbb{R}^{m}$ and $\mathbf{y} \in \mathbb{R}^{p}$ can be decomposed as described below.

Lemma 1. Call $\Pi_{\mathbf{P} \mid \mathbf{R}^{\perp}}\left(\Pi_{\mathbf{Q} \mid \mathbf{S}^{\perp}}\right)$ the projector on the model subspace $S_{M X} \equiv 179$ $\operatorname{Span}\{\mathbf{P}\} \subseteq \mathbb{R}^{m}\left(S_{M Y} \equiv \operatorname{Span}\{\mathbf{Q}\} \subseteq \mathbb{R}^{p}\right)$, along the residual subspace $S_{R X} \equiv 180$ $\operatorname{Span}\{\mathbf{R}\}^{\perp}\left(S_{R Y} \equiv \operatorname{Span}\{\mathbf{S}\}^{\perp}\right)$. Then:

$$
\begin{array}{ll}
\Pi_{\mathbf{P} \mid \mathbf{R}^{\perp}}=\mathbf{P R}^{\prime}, & \Pi_{\mathbf{R}^{\perp} \mid \mathbf{P}}=\mathbf{I}-\mathbf{P R}^{\prime}, \\
\Pi_{\mathbf{Q} \mid \mathbf{S}^{\perp}}=\mathbf{Q} \mathbf{S}^{\prime}, & \Pi_{\mathbf{S}^{\perp} \mid \mathbf{Q}}=\mathbf{I}-\mathbf{Q} \mathbf{S}^{\prime},
\end{array}
$$


where ${ }^{\perp}$ denotes the orthogonal complement of the subspace (see Proof 1 in Appendix A).

From Lemma 1, we propose the following theorem on the PLSdecomposition.

Theorem 1. The predictor and response vectors can be decomposed (by PLSR) in complementary oblique projections as:

$\mathbf{x}=\widehat{\mathbf{x}}+\widetilde{\mathbf{x}} \in \mathbb{R}^{m}$,

$\widehat{\mathbf{x}}=\mathbf{P R}^{\prime} \mathbf{x} \in S_{M X} \equiv \operatorname{Span}\{\mathbf{P}\}$,

$\widetilde{\mathbf{x}}=\left(\mathbf{I}-\mathbf{P R}^{\prime}\right) \mathbf{x} \in S_{R X} \equiv \operatorname{Span}\{\mathbf{R}\}^{\perp}$

$\mathbf{y}=\widehat{\mathbf{y}}^{*}+\widetilde{\mathbf{y}}_{2} \in \mathbb{R}^{p}$,

$\widehat{\mathbf{y}}^{*}=\mathbf{Q} \mathbf{S}^{\prime} \mathbf{y} \in S_{M Y} \equiv \operatorname{Span}\{\mathbf{Q}\}$,

$\widetilde{\mathbf{y}}_{2}=\left(\mathbf{I}-\mathbf{Q} \mathbf{S}^{\prime}\right) \mathbf{y} \in S_{R Y} \equiv \operatorname{Span}\left\{\mathbf{S}^{\perp}\right.$,

$\widehat{\mathbf{y}}^{*}=\widehat{\mathbf{y}}+\widetilde{\mathbf{y}}_{1} \in S_{M Y}$

$\widehat{\mathbf{y}}=\mathbf{Q B R}^{\prime} \widehat{\mathbf{x}} \in S_{M Y}$,

$\widetilde{\mathbf{y}}_{1}=\mathbf{Q S} \mathbf{y}-\mathbf{Q B R}^{\prime} \mathbf{x} \in S_{M Y}$,

where $\widetilde{\mathbf{y}}_{1}$ is the error of the linear transformation $\widehat{\mathbf{x}} \rightarrow \widehat{\mathbf{y}}^{*}$, and $\widehat{\mathbf{y}}$ denotes the predictable part of $\widehat{\mathbf{y}}^{*}$ from $\widehat{\mathbf{x}}$ (see Proof 2 in Appendix $\mathrm{A}$ ).

The minimum angle $\theta_{X}$ between the two complementary subspaces $S_{M X}$ and $S_{R X}$ is defined as the number $0 \leq \theta_{X} \leq \pi / 2$ that satisfies: $\cos \theta_{X}=\max \left(\widehat{\mathbf{x}}^{\prime} \widetilde{\mathbf{x}} /\|\widehat{\mathbf{x}}\|\|\widetilde{\mathbf{x}}\|\right)$. A similar definition applies for the minimum angle $\theta_{Y}$ between $S_{M Y}$ and $S_{R Y}$. In turn, $\theta_{X}\left(\theta_{Y}\right)$ can be estimated through [28]:

$\sin \theta_{X}=1 /\left\|\mathbf{P R}^{\prime}\right\|_{2}, \quad \sin \theta_{Y}=1 /\left\|\mathbf{Q} \mathbf{S}^{\prime}\right\|_{2}$

The coordinates of $\widehat{\mathbf{x}}$ and $\widehat{\mathbf{y}}^{*}$ on the model subspaces $S_{M X}$ and $S_{M Y}$, are given by:

$\mathbf{t}=\mathbf{R}^{\prime} \mathbf{x}, \quad \mathbf{u}=\mathbf{S}^{\prime} \mathbf{y}$,

where $\mathbf{t}=\left[t_{1} \cdots t_{A}\right]^{\prime}$ and $\mathbf{u}=\left[u_{1} \cdots u_{A}\right]^{\prime}$. The latent vectors are related by:

$\mathbf{u}=\mathbf{B t}+\widetilde{\mathbf{u}}$

where $\widehat{\mathbf{u}}=\mathbf{B t}=\mathbf{S}^{\prime} \widehat{\mathbf{y}}$ is the prediction of $\mathbf{u}$. Then, from Eqs. (10)-(12), (14a) it results:

$\widehat{\mathbf{x}}=\mathbf{P t}, \quad \widehat{\mathbf{y}}=\mathbf{Q} \widehat{\mathbf{u}}$.
Therefore, the corresponding correlation matrices are related as 215 follows:

$$
\begin{aligned}
& \boldsymbol{\Lambda}=\mathbf{R}^{\prime} \mathbf{R}_{\mathbf{x}} \mathbf{R}=(N-1)^{-1} \mathbf{T}^{\prime} \mathbf{T}=\operatorname{diag}\left(\lambda_{1} \ldots \lambda_{A}\right), \\
& \boldsymbol{\Delta}=(N-1)^{-1} \widehat{\mathbf{U}}^{\prime} \widehat{\mathbf{U}}=\mathbf{B} \mathbf{\Lambda} \mathbf{B}=\operatorname{diag}\left(\widehat{\delta}_{1} \ldots \widehat{\delta}_{A}\right), \\
& \mathbf{R}_{\widehat{\mathbf{x}}}=(N-1)^{-1} \widehat{\mathbf{X}}^{\prime} \widehat{\mathbf{X}}=\mathbf{P} \mathbf{\Lambda} \mathbf{P}^{\prime}=\sum_{a=1}^{A} \lambda_{a} \mathbf{p}_{a} \mathbf{p}_{a}^{\prime}, \\
& \mathbf{R}_{\widehat{\mathbf{y}}}=(N-1)^{-1} \widehat{\mathbf{Y}}^{\prime} \widehat{\mathbf{Y}}=\mathbf{Q} \Delta \mathbf{Q}^{\prime}=\sum_{a=1}^{A} \lambda_{a} b_{a}^{2} \mathbf{q}_{a} \mathbf{q}_{a}^{\prime},
\end{aligned}
$$

where $\lambda_{i}$ and $\widehat{\delta}_{i}$ are the estimated variances of $t_{i}$ and $\widehat{u}_{i}$, respectively. 223

The main geometric properties of the PLS-decomposition are repre- 225 sented in Fig. 1 for an idealized low-order hypothetical system. Both 226 measurement vectors ( $\mathbf{x}$ and $\mathbf{y}$ ) are decomposed into their respective 227 projections; and the corresponding control regions (intervals for $S_{R X} 228$ and $S_{R Y}$, and ellipses for $S_{M X}$ and $S_{M Y}$ ), are also indicated. The oblique 229 minimum angles $\theta_{X}\left(\theta_{Y}\right)$ between the model subspace $S_{M X}\left(S_{M Y}\right)$ and 230 the complementary residual subspace $S_{R X}\left(S_{R Y}\right)$ are easily visualized. 231

\section{Process monitoring based on PLS-decomposition}

The SPM includes three main activities: 1) the detection of an out-of- 233 control condition or occurrence of an anomaly; 2) the diagnostic achieved 234 by classifying the type of anomaly that has generated the abnormal be- 235 havior, and 3) the isolation of the disturbed variables and - ideally - of 236 the disturbing sources. In the following, every one of these points is 237 analyzed.

\subsection{PLS-based fault detection}

239

Once an in-control PLSR model is developed using process data 240 under normal operating conditions, the process state can be followed 241 by projecting the actual measurements $\mathbf{x}$ and $\mathbf{y}$ on subspaces $S_{M X}, S_{R X}, 242$ $S_{M Y}$, and $S_{R Y}$, and by using the proper statistics with its control limits. 243 For example, a signal of an unexpected change in the input variables $\mathbf{X} 244$ can be detected by using the t-based Hotelling's $T^{2}$ statistic in $S_{M X}, \quad 245$

$T_{\mathbf{t}}^{2}=\left\|\boldsymbol{\Lambda}^{-1 / 2} \mathbf{t}\right\|^{2}=\left\|\left(\boldsymbol{\Lambda}^{-1 / 2} \mathbf{R}^{\prime}\right) \widehat{\mathbf{x}}\right\|^{2}=\left\|\left(\boldsymbol{\Lambda}^{-1 / 2} \mathbf{R}^{\prime}\right) \mathbf{x}\right\|^{2}$,

which can be compared against a control limit. This statistic measures a 248 meaningful change on the model subspace $S_{M X}$, and serves for discrim- 248 inating excessive changes from normal variations.

However, when a special event that was not originally considered by 250 the PLSR model occurs, the new observations $\mathbf{x}$ will partially move 251

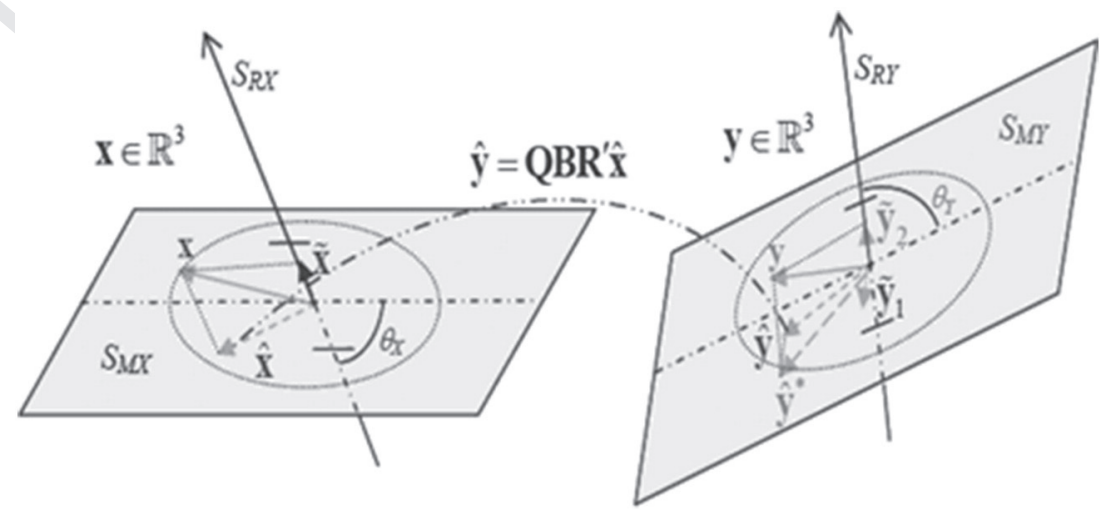

Fig. 1. Schematic representation of the PLS-decomposition of the measurement vectors $\mathbf{x}$ and $\mathbf{y}$ in their projections on the model and residue subspaces. The in-control regions are also indicated. 
outside $S_{M X}$ towards $S_{R X}$, showing an increment in the squared of the predicted error $S P E_{\mathbf{x}}$,

$S P E_{\mathbf{x}}=\|\widetilde{\mathbf{x}}\|^{2}=\left\|\left(\mathbf{I}-\mathbf{P R}^{\prime}\right) \mathbf{x}\right\|^{2}$.

This last statistic measures the distance to the model lying in $S_{R x}$; in other words, it shows a departure of $\mathbf{x}$ from the regular expected behavior by going to the subspace $S_{R x}$. Similarly, the $\widehat{\mathbf{u}}$-based Hotelling's $T^{2}$ statistic can be written as

$T_{\widehat{\mathbf{u}}}^{2}=\left\|\Delta^{-1 / 2} \widehat{\mathbf{u}}\right\|^{2}=\left\|\left(\Delta^{-1 / 2} \mathbf{S}^{\prime}\right) \widehat{\mathbf{y}}\right\|^{2}$,

and used for detecting significant amplitude changes lying in the model subspace $S_{M Y}$. Also, the distance to the regression model in $S_{M Y}$ can be written as [see Eqs. $(12,14)]$ :

$S P E_{\mathbf{y} 1}=\left\|\widetilde{\mathbf{y}}_{1}\right\|^{2}=\left\|\left[\begin{array}{ll}\mathbf{Q S}^{\prime} & -\mathbf{Q B R}^{\prime}\end{array}\right] \quad\left[\begin{array}{l}\mathbf{y} \\ \mathbf{x}\end{array}\right]\right\|^{2}=\left\|\mathbf{Q}\left(\mathbf{S}^{\prime} \mathbf{y}-\mathbf{B R}^{\prime} \mathbf{x}\right)\right\|^{2}$.

Similarly, the distance to the model lying in $S_{R Y}$ is defined as:

$S P E_{\mathbf{y} 2}=\left\|\widetilde{\mathbf{y}}_{2}\right\|^{2}=\left\|\left(\mathbf{I}-\mathbf{Q} \mathbf{S}^{\prime}\right) \mathbf{y}\right\|^{2}$

Matrices $\mathbf{R}_{\widehat{\mathbf{x}}}$ and $\mathbf{R}_{\widehat{\mathbf{y}}}$ (Eqs. 15c, 15d) are often singular because $\widehat{\mathbf{x}}$ and $\widehat{\mathbf{y}}$ typically have collinear variables, as it is inferred from Eq. (14c). Consequently, the generalized Mahalanobis distance of $\widehat{\mathbf{x}}$ and $\widehat{\mathbf{y}}$ can be used to measure the projections as follows:

$D_{\widehat{\mathbf{x}}}=\widehat{\mathbf{x}}^{\prime} \mathbf{R}_{\mathbf{x}}^{-} \widehat{\mathbf{x}}$

$D_{\widehat{\mathbf{y}}}=\widehat{\mathbf{y}}^{\prime} \mathbf{R}_{\mathbf{y}}^{-} \widehat{\mathbf{y}}$

The following theorem states that not all these statistics are independent.

Theorem 2. The Mahalanobis distances computed from $\widehat{\mathbf{x}}, \mathbf{t}, \widehat{\mathbf{u}}$, and $\widehat{\mathbf{y}}$ are equivalent, i.e., $D_{\widehat{\mathbf{x}}}=T_{\mathbf{t}}^{2}=T_{\widehat{\mathbf{u}}}^{2}=D_{\widehat{\mathbf{y}}}$ (see Proof 3 in Appendix A).

The identity in Theorem 2 suggests that the behavior of the response variables $\mathbf{y}$ can be monitored through the predictor variables $\mathbf{x}$. Therefore, the monitoring of the complete measurement space can be implemented through the following independent statistics: $T_{\mathbf{t}}^{2}, S P E_{\mathbf{x}}$, $S P E_{\mathbf{y} 1}$, and $S P E_{\mathbf{y} 2}$, each one actuating in a different subspace: $S_{M X}, S_{R X}$, $S_{M Y}$, and $S_{R Y}$, respectively (see Fig. 1). Consequently, a combined detection index $I_{T C}$ can be defined to attain the whole measurement space without signal superposition,

$I_{T C}=\frac{T_{\mathbf{t}}^{2}}{\tau_{\alpha}^{2}}+\frac{S P E_{\mathbf{x}}}{\delta_{\mathbf{x}, \alpha}^{2}}+\frac{S P E_{\mathbf{y} 1}}{\delta_{\mathbf{y} 1, \alpha}^{2}}+\frac{S P E_{\mathbf{y} 2}}{\delta_{\mathbf{y} 2, \alpha}^{2}}=\left[\begin{array}{ll}\mathbf{y}^{\prime} & \mathbf{x}^{\prime}\end{array}\right] \Phi\left[\begin{array}{l}\mathbf{y} \\ \mathbf{x}\end{array}\right]$

where $\tau_{\alpha}^{2}, \delta_{\mathbf{x}, \alpha}^{2}, \delta_{\mathbf{y} 1, \alpha}^{2}, \delta_{\mathbf{y} 2, \alpha}^{2}$ are the respective control limits with a confidence level $\alpha$, and $\Phi$ is a symmetric positive-definite matrix $(\Phi>0)$ ). Note that in this way the significance level of each normalized statistic is 1 . The vector arrangement on the right of Eq. (22) is derived from Eqs. (16, 17, 19, 20), and it shows that the resulting quadratic index depends on the extended vector $\left[\mathbf{y}^{\prime} \mathbf{x}^{\prime}\right]^{\prime}$. As indicated in Appendix B, a control limit $I_{\alpha}$ (with a confidence level $\alpha$ ) can also be defined for $I_{T C}$; and this index is useful for simultaneous monitoring of product quality, process changes, and sensor problems. This combined detection index defines a multidimensional elliptic region that is compatible with the assumption of multi-normal data. Therefore, the amount of false alarms and undetected faults are significantly reduced with respect to the performance typically yielded by separated indexes [12]. The main difference among the statistics composing the combined index $I_{T C}$ are the different scaling factor and the subspace where they are relevant. Typically, the scale $\tau_{\alpha}^{2}$ for the statistic $T_{\mathbf{t}}^{2}$ is much larger than the scale factors $\delta_{\mathbf{x}, \alpha}^{2}, \delta_{\mathbf{y} 1, \alpha}^{2}, 306$ or $\delta_{\mathbf{y} 2, \alpha}^{2}$ for the quadratic errors. This happens because the noise-like 307 variability of the errors are much smaller than the signal variability 308 of $T_{\mathbf{t}}{ }^{2}$ responding to the correlation structure. Thus, a multivariate 309 change in $\mathbf{x}$ (from its normal values) that lies on $S_{M X}$ produces a con- 310 tribution to $I_{T C}$ that is lower than a change of similar magnitude on 311 $S_{R X}, S_{M Y}$, or $S_{R Y}$, measured by $S P E_{\mathbf{x}}, S P E_{\mathbf{y} 1}$, or $S P E_{\mathbf{y} 2}$, respectively.

\subsection{Diagnosis of process anomalies}

In order to determine the discriminating capacity of the four statis- 314 tics composing the index $I_{T C}$, an artificial process system - here identi- 315 fied with the subscript 0 - is created for generating ideal data obeying 316 to a predetermined correlation structure. This artificial process is de- 317 fined by first setting values to the PLSR matrices $\boldsymbol{\Lambda}_{0}, \mathbf{P}_{0}, \mathbf{B}_{0}$, and $\mathbf{Q}_{0}, 318$ that stand for a model of the system behavior under normal condition. 319 In this procedure, the random score vector $\mathbf{t}_{0} \in \mathbb{R}^{A} \sim N\left(\mathbf{0}, \boldsymbol{\Lambda}_{0}\right)$ works as 320 an independent variable to generate the associate vectors of "input" 321 and "output" responses by

$\mathbf{x}_{0}=\mathbf{P}_{0} \mathbf{t}_{0} \in S_{M X} \equiv \operatorname{Span}\left\{\mathbf{P}_{0}\right\} \subseteq \mathbb{R}^{m}$,

$\mathbf{y}_{0}=\mathbf{Q}_{0} \mathbf{B}_{0} \mathbf{t}_{0} \quad \in S_{M Y} \equiv \operatorname{Span}\left\{\mathbf{Q}_{0}\right\} \subseteq \mathbb{R}^{p}$,

Since Eq. (23) are linear combinations of random variables, the 325 resulting input and output vectors respectively follow: $\mathbf{x}_{0} \sim N 326$ $\left(\mathbf{0}, \mathbf{P}_{0} \boldsymbol{\Lambda}_{0} \mathbf{P}^{\prime}{ }_{0}\right)$ and $\mathbf{y}_{0} \sim N\left(\mathbf{0}, \mathbf{Q}_{0} \mathbf{B}_{0} \boldsymbol{\Lambda}_{0} \mathbf{B}_{0} \mathbf{Q}^{\prime}{ }_{0}\right)$, thus featuring 'common- 327 cause' variations only. Since these data stand for an ideal perfect 328 model, the residuals $\widetilde{\mathbf{x}}, \widetilde{\mathbf{y}}_{1}$ and $\widetilde{\mathbf{y}}_{2}$ are null, and there are no differences 329 between model predictions and ideal data, i.e., $\widehat{\mathbf{x}}=\mathbf{x}_{0}$ and $\widehat{\mathbf{y}}=\widehat{\mathbf{y}}^{*}=\mathbf{y}_{0} .330$

An anomalous event is detected by a significant change in the mea- 331 surements, which triggers the alarm condition when $I_{T C} \geq I_{\alpha}$. The above 332 measurement decomposition allows the discrimination among six differ- 333 ent classes of anomaly sources, facilitating in this way the diagnostic task. 334 Each one of these six alternatives is analyzed by assuming that data are 335 generated under localized pure disturbances and then observing the de- 336 tection track followed with the available model $\left(\boldsymbol{\Lambda}_{0}, \mathbf{P}_{0}, \mathbf{B}_{0}, \mathbf{Q}_{0}\right)$. The sketch 337 in Fig. 2 helps to visualize how a warning signal (at $\widetilde{\mathbf{x}}, \widetilde{\mathbf{y}}_{1}, \widetilde{\mathbf{y}}_{2}$ or $\mathbf{t}$ ) is 338 generated as the disturbed measurements ( $\mathbf{x}$ or $\mathbf{y}$ ) go through the PLSR 339 model.

Hence, under the above framework, the following classes of anoma- 341 lies are discriminated:

Class 1. Sensor faults associated to $\mathbf{X}$, represented by additional signals 343 $\xi$, that take the $\mathbf{x}$ vector out of the pattern cast by the PLSR model. In this 344 case, the input vector can be written as,

\section{$\mathbf{x}=\mathbf{x}_{0}+\boldsymbol{\xi}, \quad$ (Data generation)}

where $\mathbf{x}_{0}$ is the "free-of-change" part of the input-vector measurement. 346 Let us assume that the disturbance $\boldsymbol{\xi}$ does not follow the correlation pat- 348 tern of $\mathbf{x}_{0}$ at all (see Eq. (23)), in other words, $\boldsymbol{\xi} \in S_{R X}$. Then, $\mathbf{t}=\mathbf{R}_{0}^{\prime} \mathbf{X}=349$ $\mathbf{R}_{0}^{\prime} \mathbf{x}_{0}+\mathbf{R}_{0}^{\prime} \boldsymbol{\xi}=\mathbf{t}_{0}+0=\mathbf{t}_{0}$, which shows that the disturbance goes 350 completely to the residual variable

$\widetilde{\mathbf{x}}=\mathbf{x}-\widehat{\mathbf{x}}=\mathbf{x}-\mathbf{P}_{0} \mathbf{t}=\mathbf{x}-\mathbf{x}_{0}=\boldsymbol{\xi} \neq 0 \in S_{R X}($ Disturbance detection $)$

Class 2. Sensors faults associated to the output variable $\mathbf{y}$,

\section{$\mathbf{y}=\mathbf{y}_{0}+\boldsymbol{\eta}, \quad$ (Data generation)}

where $\mathbf{y}_{0}$ is the "free-of-change" part with a population structure deter- 356 mined by the normal conditions only. This disturbance is analyzed by 357 assuming that $\eta \in S_{R Y}$. Hence, the disturbance track from generation 358 to detection is as follows:

$\widehat{\mathbf{y}}^{*}=\mathbf{Q}_{0} \mathbf{S}_{0}^{\prime} \mathbf{y}=\mathbf{Q}_{0} \mathbf{S}_{0}^{\prime} \mathbf{y}_{0}+\mathbf{Q}_{0} \mathbf{S}_{0}^{\prime} \boldsymbol{\eta}=\mathbf{y}_{0}+0=\mathbf{y}_{0}$ 


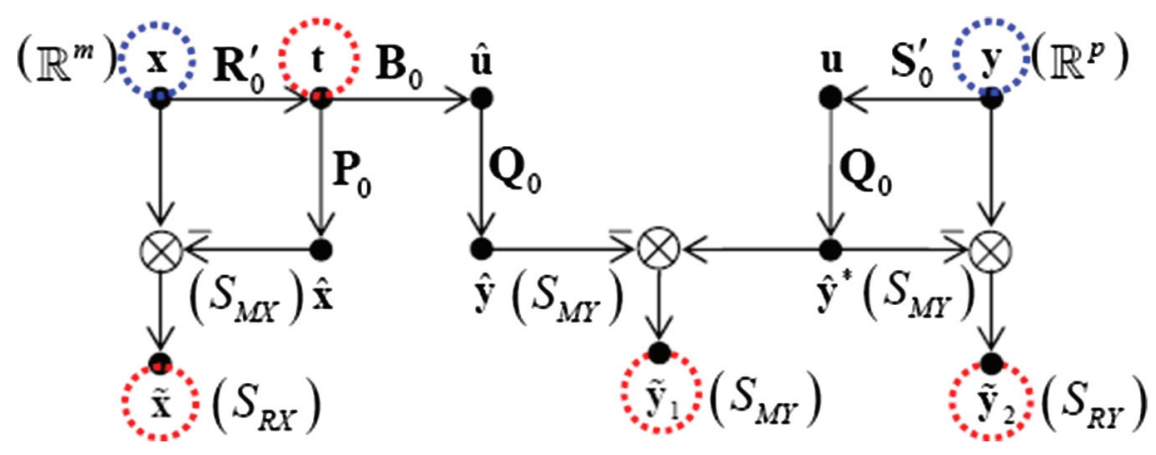

Fig. 2. Measurements decomposition based on projections onto the subspaces created by a PLSR model.

\section{$\widetilde{\mathbf{y}}_{2}=\mathbf{y}-\widehat{\mathbf{y}}^{*}=\eta \neq 0 \in S_{R Y},($ Disturbance detection $)$}

and again, a measurement disturbance not associable with the normal behavior is sent to a residual subspace, in this case, $S_{R Y}$.

Class 3. Changes in the correlation structure of $\mathbf{x}$. An $\mathbf{x}$ vector population affected by this kind of change can also be generated by the same score $\mathbf{t}_{0}$, but using an "unknown" change $\Delta \mathbf{P}=\mathbf{P}-\mathbf{P}_{0}$, i.e.,

$\mathbf{X}=\mathbf{P} \mathbf{t}_{0}=\left(\mathbf{P}_{0}+\Delta \mathbf{P}\right) \mathbf{t}_{0}=\mathbf{x}_{0}+\Delta \mathbf{P} \mathbf{t}_{0} . \quad($ Data generation $)$

It should be noted, however, that when using the measurement $\mathbf{x}$ with the available model $\mathbf{P}_{0}$, the scores change to $\mathbf{t}=\mathbf{R}_{0}^{\prime} \mathbf{X}=\mathbf{R}_{0}^{\prime}\left(\mathbf{P}_{0}+\Delta \mathbf{P}\right)$ $\mathbf{t}_{0}=\mathbf{t}_{0}+\mathbf{R}_{0}^{\prime} \Delta \mathbf{P} \mathbf{t}_{0}$, thus generating the following no-null residual values

$$
\begin{aligned}
\widetilde{\mathbf{x}} & =\mathbf{x}-\widehat{\mathbf{x}}=\left(\mathbf{x}_{0}+\Delta \mathbf{P} \mathbf{t}_{0}\right)-\mathbf{P}_{0} \mathbf{t}=\left(\mathbf{x}_{0}+\Delta \mathbf{P} \mathbf{t}_{0}\right)-\left(\mathbf{x}_{0}+\mathbf{P}_{0} \mathbf{R}_{0}^{\prime} \Delta \mathbf{P} \mathbf{t}_{0}\right) \\
& =\left(\mathbf{I}-\mathbf{P}_{0} \mathbf{R}_{0}^{\prime}\right) \Delta \mathbf{P t} \mathbf{t}_{0} \neq 0 \in S_{R X}, \widetilde{\mathbf{y}}_{1}=\widehat{\mathbf{y}}^{*}-\widehat{\mathbf{y}}=\mathbf{y}_{0}-\mathbf{Q}_{0} \mathbf{B}_{0} \mathbf{t} \\
& =\mathbf{y}_{0}-\mathbf{Q}_{0} \mathbf{B}_{0}\left(\mathbf{t}_{0}+\mathbf{R}_{0}^{\prime} \Delta \mathbf{P} \mathbf{t}_{0}\right)=-\mathbf{Q}_{0} \mathbf{B}_{0} \mathbf{R}_{0}^{\prime} \Delta \mathbf{P t}_{0} \neq 0 \in S_{M Y} .
\end{aligned}
$$

where the residues $\widetilde{\mathbf{x}}$ and $\widetilde{\mathbf{y}}_{1}$ are used to detect the disturbance. Also, it is important to note that $\left\|\boldsymbol{\Lambda}_{0}^{-1 / 2} \mathbf{R}_{0}^{\prime} \widehat{\mathbf{x}}\right\|^{2} / \tau_{\alpha}^{2} \ll\|\widetilde{\mathbf{x}}\|^{2} / \delta_{\mathbf{x}, \alpha}^{2}$ when $\|\widehat{\mathbf{x}}\|^{2}=\|\widetilde{\mathbf{x}}\|^{2}$.

Class 4. Changes in the intrinsic gains. The diagonal matrix $\mathbf{B}$ is the core place where the PLSR model ties up input with output variables. Let us assume an unknown change occurred in this matrix, i.e.,

$\widehat{\mathbf{y}}^{*}=\mathbf{Q}_{0} \mathbf{B t}_{0}=\mathbf{Q}_{0}\left(\mathbf{B}_{0}+\Delta \mathbf{B}\right) \mathbf{t}_{0}=\mathbf{y}_{0}+\mathbf{Q}_{0} \Delta \mathbf{B} \mathbf{t}_{0}$, (Data generation $)$

and analyze the effects on the statistics being considered for monitoring,

$\widetilde{\mathbf{y}}_{1}=\widehat{\mathbf{y}}^{*}-\widehat{\mathbf{y}}=\left(\mathbf{y}_{0}+\mathbf{Q}_{0} \Delta \mathbf{B t}_{0}\right)-\mathbf{Q}_{0} \mathbf{B}_{0} \mathbf{t}_{0}=\mathbf{Q}_{0} \Delta \mathbf{B} \mathbf{t}_{0} \neq 0 \in S_{M Y}$

which tells that the "detecting signal" should be observed by monitoring the residue $\widetilde{\mathbf{y}}_{1}$ on $S_{M Y}$, while the remaining statistics remain unaffected (see Fig. 2).

Class 5. Changes in the correlation structure of $\mathbf{y}$. Let us assume an unknown change in the matrix $\mathbf{Q}$ i.e.

$\mathbf{y}=\mathbf{Q B}_{0} \mathbf{t}_{0}=\left(\mathbf{Q}_{0}+\Delta \mathbf{Q}\right) \mathbf{B}_{0} \mathbf{t}_{0}=\mathbf{y}_{0}+\Delta \mathbf{Q} \mathbf{B}_{0} \mathbf{t}_{0} \quad$ (Data generation)

and the detection track characterized by

$$
\begin{aligned}
\widehat{\mathbf{y}}^{*} & =\mathbf{Q}_{0} \mathbf{S}_{0}^{\prime} \mathbf{y}=\mathbf{Q}_{0} \mathbf{S}_{0}^{\prime}\left(\mathbf{y}_{0}+\Delta \mathbf{Q} \mathbf{B}_{0} \mathbf{t}_{0}\right)=\mathbf{y}_{0}+\mathbf{Q}_{0} \mathbf{S}_{0}^{\prime} \Delta \mathbf{Q} \mathbf{B}_{0} \mathbf{t}_{0} \widetilde{\mathbf{y}}_{2}=\mathbf{y}-\widehat{\mathbf{y}}^{*} \\
& =\left(\mathbf{y}_{0}+\Delta \mathbf{Q} \mathbf{B}_{0} \mathbf{t}_{0}\right)-\left(\mathbf{y}_{0}+\mathbf{Q}_{0} \mathbf{S}_{0} \Delta \mathbf{Q} \mathbf{B}_{0} \mathbf{t}_{0}\right) \\
& =\left(\mathbf{I}-\mathbf{Q}_{0} \mathbf{S}^{\prime}{ }_{0}\right) \Delta \mathbf{Q} \mathbf{B}_{0} \mathbf{t}_{0} \neq 0 \in S_{R Y}
\end{aligned}
$$

and also

$\widetilde{\mathbf{y}}_{1}=\widehat{\mathbf{y}}^{*}-\widehat{\mathbf{y}}=\left(\mathbf{y}_{0}+\mathbf{Q}_{0} \mathbf{S}_{0}^{\prime} \Delta \mathbf{Q} \mathbf{B}_{0} \mathbf{t}_{0}\right)-\mathbf{y}_{0}=\mathbf{Q}_{0} \mathbf{S}_{0}^{\prime} \Delta \mathbf{Q} \mathbf{B}_{0} \mathbf{t}_{0} \neq 0 \in S_{M Y}$.

where the residues $\widetilde{\mathbf{y}}_{1}$ and $\widetilde{\mathbf{y}}_{2}$ are used to "detect the disturbance".

Class 6. A significant change in the process condition produces 395 measurements that follow the correlation structure captured by the 396 undisturbed PLSR model. This type of disturbance can be represented 397 by changes in the population parameters of $\mathbf{t}_{0} \sim N\left(\mathbf{0}, \boldsymbol{\Lambda}_{0}\right)$, i.e., we have 398 to assume a displacement of $E\left\{\mathbf{t}_{0}\right\}$ from $\mathbf{0}$ to a $\boldsymbol{\mu}_{\mathbf{t}} \neq \mathbf{0}$, or a significant 399 change in the variability from $\boldsymbol{\Lambda}_{0}$ to $\boldsymbol{\Lambda}_{\mathbf{t}} \neq \boldsymbol{\Lambda}_{\mathbf{0}}$. Hence, the anomaly in 400 this case produces

$\mathbf{t}=\mathbf{t}_{0}+\Delta \mathbf{t} \sim N\left(\boldsymbol{\mu}_{\mathbf{t}}, \boldsymbol{\Lambda}_{\mathbf{t}}\right) \quad$ (Data generation)

with a $\Delta \mathbf{t}$ of magnitude such that: $T_{\mathbf{t}}^{2}=\left\|\boldsymbol{\Lambda}_{0}^{-1 / 2} \mathbf{t}\right\|^{2}=\| \boldsymbol{\Lambda}_{0}^{-1 / 2}\left(\mathbf{t}_{0}+40 z\right.$ $\Delta \mathbf{t}) \|^{2} \geq \tau_{\alpha}^{2}$ (disturbance detection).

404

The above results are summarized in Table 2. The highlighted dis- 405 crimination patterns indicate the statistics that are activated as soon 406 as the measurements $(\mathbf{x}, \mathbf{y})$ bring information about a localized model 407 mismatch. The six analyzed anomalies can qualitatively be grouped 408 into the following three categories: sensor fault (classes 1 and 2), pro- 409 cess change (classes 3, 4 and 5), and excessive (quality) operation 410 change (class 6).

Some final comments about the effect of the dimension $A$ on the dis- 412 criminating sensitivity seem convenient at this point. Recall that the 413 predictor variables $\mathbf{X}$ are assumed as belonging to an $m$ dimensional 414 space, i.e., $\mathbf{x} \in \mathbb{R}^{m}$. This space is then projected onto the subspaces $S_{M X} 415$ and $S_{R X}$ where $\operatorname{dim}\left(S_{M X}\right)=A \leq m$ and $\operatorname{dim}\left(S_{R X}\right)=m$-A. Hence, when 416 $A \rightarrow m$, the subspace $S_{R X}$ (useful for detecting sensor faults in the predic- 417 tor variables $\mathbf{x}$ ) reduces, leaving information of possible anomalous 418 measurements in $S_{M X}$. It is then understandable that when $A$ increases 419 beyond a certain point, the ability for failure detection on $S_{R X}$ tends to 420 disappear. At the same time, too small values of $A$ might move the cor- 421 related behavior to $S_{R X}$, playing an interfering roll in the residue index 422 $S P E_{\mathbf{x}}$. A similar reasoning can be done with the output variables $\mathbf{y}$, orig- 423 inally in a space of dimension $p$, i.e., $\mathbf{y} \in \mathbb{R}^{p}$.

Table 2

Expected statistics pattern for each anomaly class.

$\mathrm{t} 2.1$ Anomaly class

Statistics composing $I_{T C}$ (associated subspaces) $\begin{array}{lllll}T_{\mathbf{t}}^{2} / \tau_{\alpha}^{2} & S P E_{\mathbf{x}} / & S P E_{\mathbf{y} 1} / & S P E_{\mathbf{y} 2} / & \mathrm{t} 2.4\end{array}$

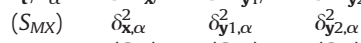

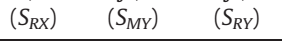

Class 1: fault of sensors in $\mathbf{x}(\boldsymbol{\xi})$ Class 2: fault of sensors in $\mathbf{y}(\boldsymbol{\eta})$ Class 3: changes in correlations of $\mathbf{x}(\Delta \mathbf{P})$ Class 4: changes in the intrinsic gains $(\Delta \mathbf{B})$ Class 5: changes in correlations of $\mathbf{y}(\Delta \mathbf{Q})$ Class 6: excessive change in $\left(\boldsymbol{\mu}_{\mathbf{t}}, \boldsymbol{\Lambda}_{\mathbf{t}}\right)$

++ : significant value. - : negligible value. 
448

450

\subsection{Isolation of disturbed variables or disturbing sources}

When trying to localize a faulty sensor or detect an abnormal correlation, the identification of the involved variables becomes very helpful. The use of contribution analysis to characterize an anomaly has been previously proposed [11]. The preliminary classification of the anomalous event according to Table 2, enable us to restrict the search problem to one or two statistics.

A simple strategy for decomposing the four statistics in Eq. (22) is also proposed here. The arrangement can be applied to any quadratic form $I^{2}=\mathbf{z}^{\prime} \mathbf{A z}$ (like $T_{t}^{2}, S P E_{\mathbf{x}}, S P E_{\mathbf{y} 1}$, or $S P E_{\mathbf{y} 2}$ ), to reveal the contribution intensity of each variable $z_{i}$. This decomposition is performed as follows:

$I^{2}=\mathbf{z}^{\prime} \mathbf{A} \sum_{i=1}^{n} \mathbf{z}_{i}=\sum_{i=1}^{n} \mathbf{z}^{\prime} \mathbf{a}_{i} z_{i}=\sum_{i=1}^{n} c I^{2}\left(z_{i}\right)$

where $\mathbf{a}_{i}=\left[\begin{array}{lll}a_{i 1} & \cdots & a_{i n}\end{array}\right]^{\prime}$ is the $i$-th column of the matrix $\mathbf{A}$, $\mathbf{z}_{i} \triangleq\left[0 \cdots z_{i} \cdots 0\right]^{\prime}(n \times 1)$ is a vector with only a nonzero value in the $i$-th position, and $\mathrm{cl}^{2}\left(z_{i}\right)$ stands for the contribution of $z_{i}$ to $I^{2}$. This contribution captures the gradient of the statistic in the $z_{i}$ direction, i.e.,

$\frac{1}{2} \nabla_{\mathbf{z}}\left(I^{2}\right) \cdot \mathbf{z}_{i}=\frac{1}{2} \nabla_{\mathbf{z}}\left(\mathbf{z}^{\prime} \mathbf{A z}\right) \cdot \mathbf{z}_{i}=\mathbf{z}^{\prime} \mathbf{a}_{i} z_{i}=c I^{2}\left(z_{i}\right)$.

Note that it can also be written in terms of the projections by substituting the relationships $\mathbf{R}^{\prime} \widehat{\mathbf{x}}=\mathbf{R}^{\prime} \mathbf{X},\left(\mathbf{I}-\mathbf{P R}^{\prime}\right) \widetilde{\mathbf{x}}=\left(\mathbf{I}-\mathbf{P} \mathbf{R}^{\prime}\right) \mathbf{X}, \mathbf{S}^{\prime} \widehat{\mathbf{y}}^{*}=\mathbf{S}^{\prime}$ $\mathbf{y}$ and $\left(\mathbf{I}-\mathbf{Q S}^{\prime}\right) \widetilde{\mathbf{y}}_{2}=\left(\mathbf{I}-\mathbf{Q} \mathbf{S}^{\prime}\right) \mathbf{y}$ in Eqs. $(16,17,19,20)$, yielding

$c T_{\mathbf{t}}^{2}\left(x_{i}\right)=\mathbf{x}^{\prime} \mathbf{a}_{i} x_{i} \equiv \widehat{\mathbf{x}}^{\prime} \mathbf{a}_{i} \widehat{x}_{i}, \quad$ with $\mathbf{A}=\mathbf{R} \boldsymbol{\Lambda}^{-1} \mathbf{R}^{\prime}=\left[\mathbf{a}_{1} \cdots \mathbf{a}_{m}\right]_{m \times m}$

$c \operatorname{SPE} E_{\mathbf{x}}\left(x_{i}\right)=\widetilde{\mathbf{x}}^{\prime} \mathbf{a}_{i} \widetilde{x}_{i}$, with $\mathbf{A}=\left(\mathbf{I}-\mathbf{P} \mathbf{R}^{\prime}\right)^{\prime}\left(\mathbf{I}-\mathbf{P R}^{\prime}\right)=\left[\mathbf{a}_{1} \cdots \mathbf{a}_{m}\right]_{m \times m}$

$$
\operatorname{CSPE}_{\mathbf{y} 1}\left(x_{i}\right)=\left[\widehat{\mathbf{y}}^{* \prime} \widehat{\mathbf{x}}^{\prime}\right] \mathbf{a}_{j} \widehat{x}_{i}, \quad \operatorname{CSPE} E_{\mathbf{y} 1}\left(y_{j}\right)=\left[\widehat{\mathbf{y}}^{* \prime} \widehat{\mathbf{x}}^{\prime}\right] \mathbf{a}_{j} \widehat{y}_{j}^{*}
$$$$
\text { with } \mathbf{A}=\left[\begin{array}{cc}
\mathbf{S S}^{\prime} & -\mathbf{S B R}^{\prime} \\
-\mathbf{R B S} & \mathbf{R B}^{2} \mathbf{R}^{\prime}
\end{array}\right]=\left[\begin{array}{lll}
\mathbf{a}_{1} \cdots \mathbf{a}_{p} \mathbf{a}_{p+1} \cdots \mathbf{a}_{p+m}
\end{array}\right]_{(p+m) \times(p+m)}
$$

$$
\operatorname{cSPE}_{\mathbf{y} 2}\left(y_{j}\right)=\widetilde{\mathbf{y}}_{2}^{\prime} \mathbf{a}_{j} \widetilde{y}_{2, j}, \text { with } \mathbf{A}=\left(\mathbf{I}-\mathbf{Q} \mathbf{S}^{\prime}\right)^{\prime}\left(\mathbf{I}-\mathbf{Q} \mathbf{S}^{\prime}\right)=\left[\mathbf{a}_{1} \cdots \mathbf{a}_{p}\right]_{p \times p} .
$$

Then, by combining Eqs. (22), (24), (26)-(29) gives

$$
\begin{aligned}
I_{T C}= & I_{M X}(\mathbf{x})+I_{R X}(\mathbf{x})+I_{R Y 1}(\mathbf{x}, \mathbf{y})+I_{R Y 2}(\mathbf{y}) \\
I_{T C}= & \sum_{i=1}^{m} c I_{M X}\left(x_{i}\right)+\sum_{i=1}^{m} c I_{R X}\left(x_{i}\right)+\left(\sum_{i=1}^{m} c I_{R Y 1}\left(x_{i}\right)+\sum_{j=1}^{p} c I_{R Y 1}\left(y_{j}\right)\right) \\
& +\sum_{j=1}^{p} c I_{R Y 2}\left(y_{j}\right)
\end{aligned}
$$

where e.g., the term $c I_{M X}\left(x_{i}\right)=c T_{t}^{2}\left(x_{i}\right) / \tau_{\alpha}^{2}$ in Eq. (30) is the normalized contribution of $x_{i}$ to $I_{T C}$ through the projection on $S_{M X}$ (see Eq. (26)). Furthermore, since $I_{M X}$ has a significance level of 1 , then the significance level of their contributions $c I_{M X}$ is also adopted equal to 1 . In particular, $c T_{\mathbf{t}}^{2}\left(x_{i}\right)=x_{i} \mathbf{a}_{i} \mathbf{x}^{\mathbf{x}}$ (Eq. (26)) is equivalent to the contribution defined by Westerhuis et al. [29]; and it tends to the contribution reported by Alvarez et al. [30], when $S_{M X} \rightarrow \mathbb{R}^{m}$, because $D_{\widehat{\mathbf{x}}} \rightarrow T_{\mathbf{x}}^{2}=\mathbf{x}^{\prime} \mathbf{R}_{\mathbf{x}}^{-1} \mathbf{x}$ with $\mathbf{R}_{\mathbf{x}}>0$.

The variable contribution defined by Eq. (24) guarantees a correct diagnosis when $\mathbf{A}$ is positive semidefinite [11], which always holds for the four statistics of Eqs. (26)-(29). A reliable isolation of faulty sensors must correctly indicate the disturbed variable. For example, a fault in the $k$-th sensor of $\mathbf{x}$ can be represented as $\mathbf{x}=\mathbf{x}_{0}+f_{k} \boldsymbol{\xi}_{k}$ [10], where $f_{k}$ is the fault magnitude and $\xi_{k}=[00 \cdots 1 \cdots 0]^{\prime}$ is the fault direction. If the faulty measurement projection is mostly included in $S_{R X}$ (see Class 1 ), then it can be expressed as: $\widetilde{\mathbf{x}} \cong f_{k} \xi_{k}$ (i.e. $\widetilde{x}_{k} \cong f_{k}$ and $\widetilde{x}_{i} \cong 0 \forall i \neq k$ ).
Thus, by including such value into the contributions to $I_{T C}$ (depending 474 on the subspace), it results:

$$
\begin{aligned}
& I_{M X}, I_{R Y 1}, I_{R Y 2} \cong 0, \\
& C I_{R X}\left(x_{i}\right)=\widetilde{\mathbf{x}}^{\prime} \mathbf{a}_{i} \widetilde{x}_{i} / \delta_{\mathbf{x}, \alpha}^{2}= \begin{cases}f_{k} \xi^{\prime}{ }_{k} \mathbf{a}_{i} 0 / \delta_{\mathbf{x}, \alpha}^{2}=0 & \text { for } i \neq k \\
f_{k} \xi^{\prime}{ }_{k} \mathbf{a}_{i} f_{k} / \delta_{\mathbf{x}, \alpha}^{2}=f_{k}^{2} a_{i i} / \delta_{\mathbf{x}, \alpha}^{2} & \text { for } i=k\end{cases}
\end{aligned}
$$

A correct diagnosis occurs when the contribution of the non-faulty 478 variable is less than or equal to the contribution of the faulty variable, 479 i.e. when $c I_{R X}\left(x_{k}\right) \geq 0$ (see Eq. (31)). Therefore, a correct isolation is 480 guaranteed when $a_{i i} \geq 0$, which is always true since $\mathbf{A} \geq 0$ (Eq. (27)). 481

\section{Simulation test}

The described monitoring procedure is now tested with random mea- 483 surements obtained from the numerical simulation of two systems of dif- 484 ferent complexity. The first system is a synthetic example representing a 485 hypothetical static process, where the internal data structure was arbi- 486 trary chosen. The second application is a more realistic problem where 487 the variables of a dynamic process under normal conditions are empiri- 488 cally modeled by PLSR.

\subsection{A four-state static process}

490

The synthetic example is first simulated to better interpret the pro- 491 posed methodology as a monitoring tool. The normal operation of the 492 chosen system includes four internal states, which are represented by 493 the following four points of its latent space (t-scores): $\left\{\left(t_{1}^{*}, t_{2}^{*}\right)\right\}_{1} \ldots 4=494$ $\{(1,1),(1,3),(3,3),(3,1)\}$. The "measurements" of the external variables, $\mathbf{x} 495$ and $\mathbf{y}$, are generated by adding zero-mean Gaussian random noises 496 $\left(\boldsymbol{\varepsilon}_{i}, i=1 \ldots 4\right)$ to the PLSR correlation structure characterized by the 497 arbitrary-selected process matrices $\mathbf{P}, \mathbf{Q}$ and $\mathbf{B}$, as follows:

$$
\begin{aligned}
& \text { Internal part } \begin{cases}\mathbf{t}_{0}=\mathbf{t}^{*}+\boldsymbol{\varepsilon}_{1}, & \boldsymbol{\varepsilon}_{1} \sim N\left(\mathbf{0}, 0.1^{2} \mathbf{I}_{2}\right), \\
\mathbf{u}=\mathbf{B t}_{0}+\boldsymbol{\varepsilon}_{2}, \quad \mathbf{B}=\operatorname{diag}(2,0.5), & \boldsymbol{\varepsilon}_{2} \sim N\left(\mathbf{0}, 0.05^{2} \mathbf{I}_{2}\right),\end{cases} \\
& \text { External part }\left\{\begin{array}{lll}
\mathbf{x}=\mathbf{P t}_{0}+\boldsymbol{\varepsilon}_{3}, & \mathbf{P}=\left[\begin{array}{ll}
\mathbf{p}_{1} & \mathbf{p}_{2}
\end{array}\right], & \boldsymbol{\varepsilon}_{3} \sim N\left(\mathbf{0}, 0.05^{2} \mathbf{I}_{7}\right), \\
\mathbf{y}=\mathbf{Q u}+\boldsymbol{\varepsilon}_{4}, & \mathbf{Q}=\left[\begin{array}{ll}
\mathbf{q}_{1} & \mathbf{q}_{2}
\end{array}\right], & \boldsymbol{\varepsilon}_{4} \sim N\left(\mathbf{0}, 0.05^{2} \mathbf{I}_{5}\right),
\end{array}\right. \\
& \mathbf{p}_{1}=[0.4045,0,0.5394,0.2697,0.1348,0,0.6742]^{\prime} \text {, } \\
& \mathbf{p}_{2}=[0,0.7906,0.1581,-0.1581,-0.3162,0.4743,0]^{\prime} \text {, } \\
& \mathbf{q}_{1}=[0.5883,0,-0.1961,0,0.7845]^{\prime}, \\
& \mathbf{q}_{2}=[0.0081,0.7054,0.0678,-0.7054,0.0109]^{\prime},
\end{aligned}
$$

390

\begin{tabular}{|c|c|c|c|}
\hline Location & Anomaly class & Magnitude of the change/fault & $\begin{array}{l}I_{T C} / I_{a} \\
\left(I_{a}=2.266\right)\end{array}$ \\
\hline$k=11$ & 1 & $\Delta \mathbf{x}=\left[\begin{array}{lllllll}0.3 & 0 & 0 & 0 & 0 & 0.25 & 0\end{array}\right]^{\prime}$ (Multiple fault) & 1.980 \\
\hline$k=19$ & 2 & $\Delta \mathbf{y}=\left[\begin{array}{lllll}0.35 & 0 & 0 & 0 & 0\end{array}\right]^{\prime}($ Single fault $)$ & 2.381 \\
\hline$k=27$ & 3 & 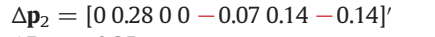 & 5.799 \\
\hline$k=35$ & 4 & $\Delta \mathbf{B}_{22}=0.25$ & 3.291 \\
\hline$k=43$ & 5 & $\Delta \mathbf{q}_{1}=\left[\begin{array}{lllll}-0.05 & 0.025 & 0.05 & 0.025 & -0.1\end{array}\right]^{\prime}$ & 3.700 \\
\hline$k=51$ & 6 & $\Delta \mathbf{t}=\left[\begin{array}{ll}0 & 6\end{array}\right]^{\prime}$ & 2.715 \\
\hline
\end{tabular}

This model is used to simulate 40 multivariate observations under 501 'normal conditions' and the generated dataset is used to fit the PLSR 502 model. The selection of $A=2$ is determined by monitoring the simulta- 503 neous deflation of $\mathbf{X}$ and $\mathbf{Y}$ [18]; in this way the errors regarding the 504 "true" matrices $\mathbf{Q} \mathbf{B}$, and $\mathbf{P}$ are negligible. The control limits $\tau_{\alpha}^{2}, \delta_{\mathbf{x}, \alpha}^{2}, 505$ $\delta_{\mathbf{y} 1, \alpha}^{2}$ and $\delta_{\mathbf{y} 2, \alpha}^{2}$ are calculated as explained in Appendix B. 506

Table 3 shows six simulated anomalies (one for each class in 507 Table 2): a) anomalies of classes 1 and 2 correspond to sensor biases 508 that are simulated by disturbing the measurements $\mathbf{x}$ and $\mathbf{y}$; b) 509

Table 3

Simulated scenarios of anomalies. 
anomalies of classes 3,4 , and 5 are implemented by altering the process matrices; and c) anomaly of class 6 consists in adding up a change $\Delta \mathbf{t}$ to $\mathbf{t}_{0}$, such that $I_{T C}>I_{\alpha}$. Each fault is simulated by affecting only one sample point at a discrete time, $k$; and immediately the anomaly is canceled from $k+1$ onwards. These anomalies represent a hard test for evaluating the ability of the method.
Fig. 3a shows the time evolution of the $I_{T C}$ detection index and its 516 component statistics. The alarm condition is triggered at a given sample 517 $k$, when the $I_{T C}$ index overpasses the control limit. The index $I_{T C}$ proved 518 to be effective for detecting all simulated anomalies. Table 3 shows the 519 proportion of the variation of $I_{T C}$ with respect to control limit for each 520 simulated fault-scenario. The patterns of the statistics recorded in 521

a)

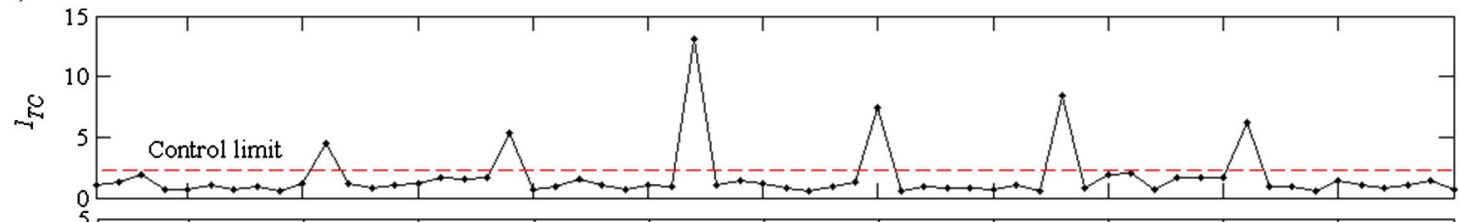

索

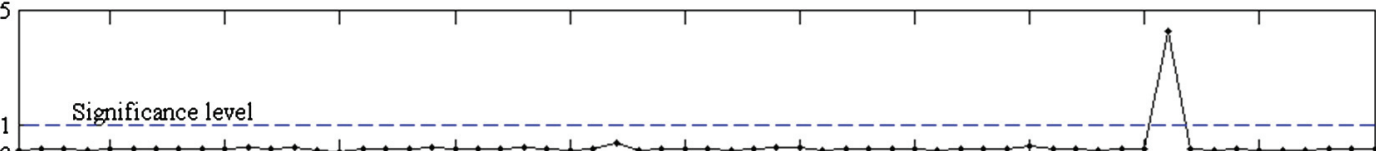

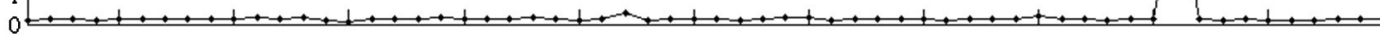
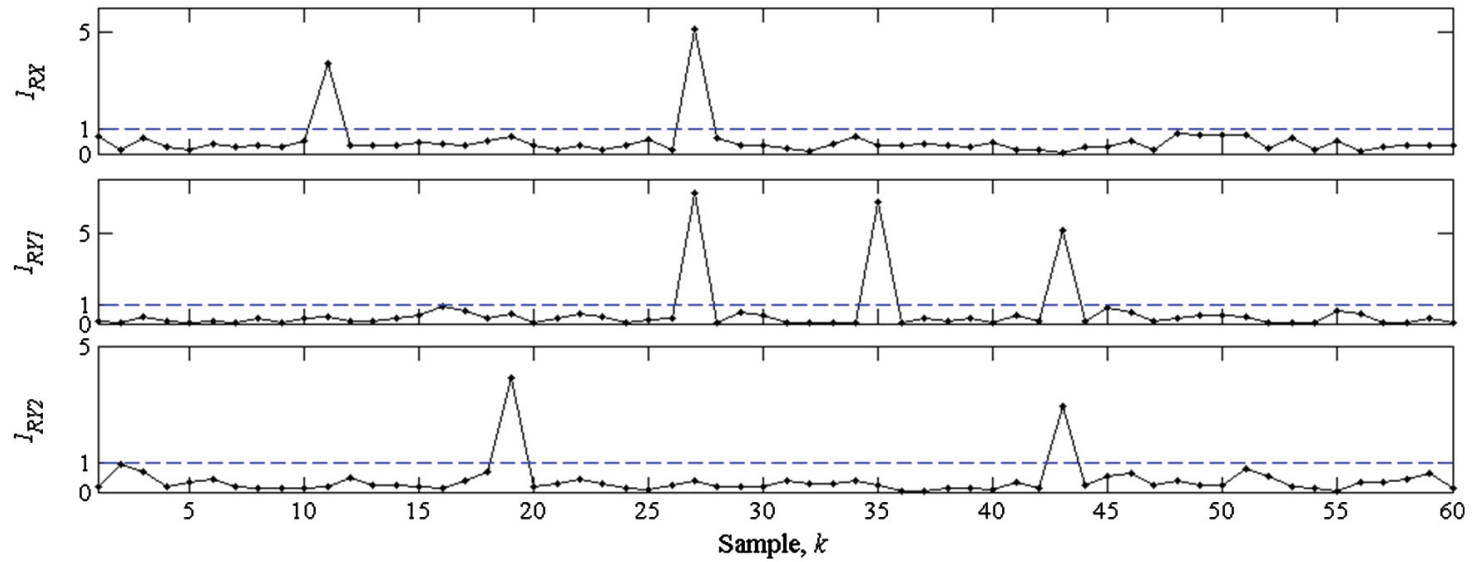

b)
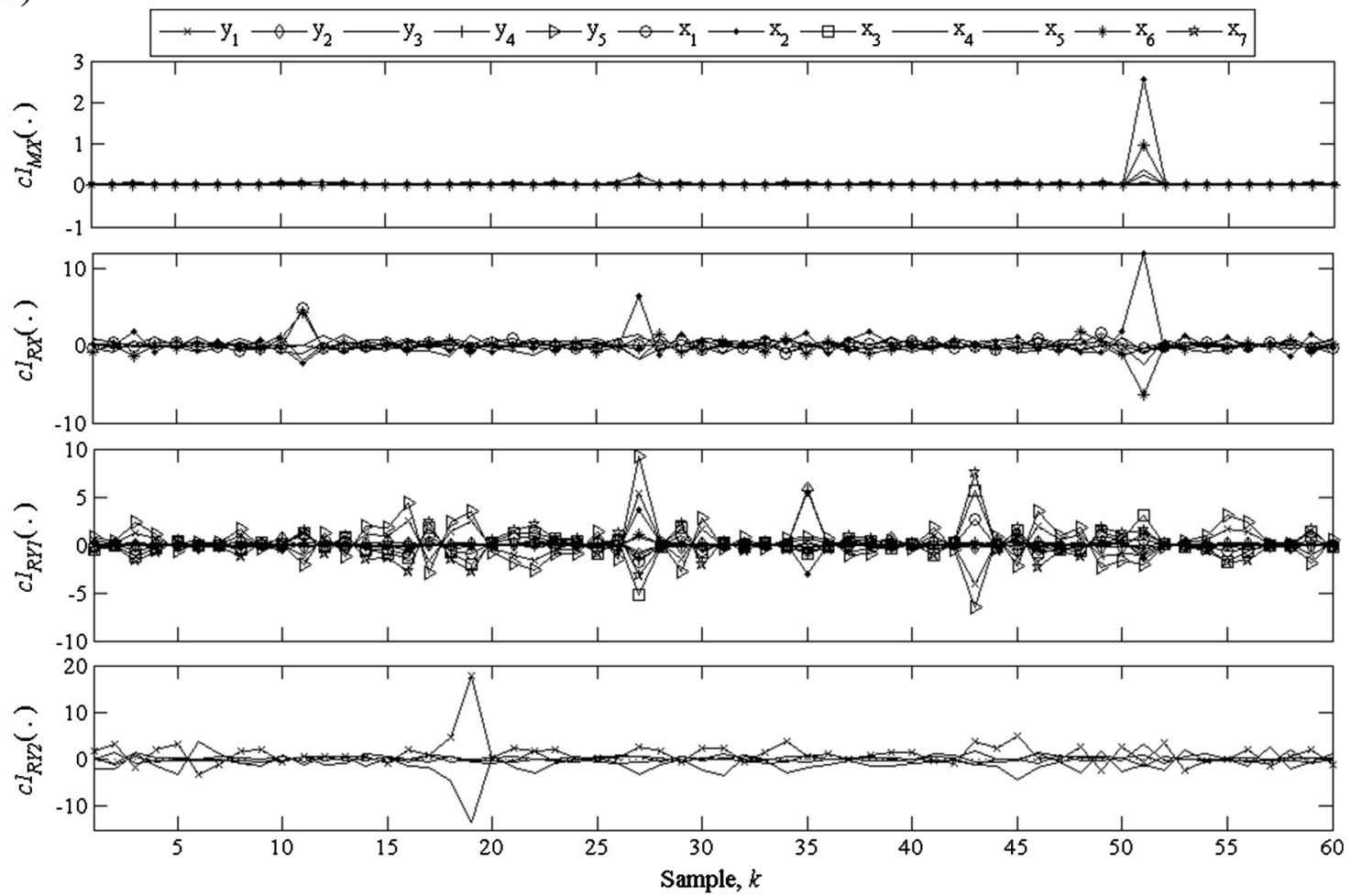

Fig. 3. Simulation example based on a statistic process. (a) Temporal evolution of the combined index $I_{T C}$ and of its component statistics. (b) Variable contributions to each component statistics of $I_{T C}$. 
Fig. 3a along with the information given in Table 2 allow an unambiguous classification of each anomaly class (see Table 4). Fig. 3b shows the instantaneous variable contributions to each component statistic of $I_{T C}$. For example, at $k=11$, the main contributions to $I_{T C}$ are $c I_{R X}\left(x_{1}\right)$ and $C I_{R X}\left(x_{6}\right)$; and therefore faults in sensors $x_{1}$ and $x_{6}$ are diagnosed (first row of Table 4$)$. Similarly, at $k=19, C I_{R Y 2}\left(y_{1}\right)$ diagnoses a fault in sensor $y_{1}$ (second row of Table 4). The remaining results in Table 4 are summarized as follows: a) at $k=27$ and $k=43$, correlation changes in $\mathbf{x}$ and $\mathbf{y}$ are respectively diagnosed, which indicate major changes in the original external correlations (i.e., $\mathbf{P}$ and $\mathbf{Q}$ in Eq. (32)); b) at $k=35, C I_{R Y 1}\left(y_{2}\right)$ and $c I_{R Y 1}\left(y_{4}\right)$ diagnose an upset of the $t_{2}-u_{2}$ inner relation, because the contributions of $y_{2}$ and $y_{4}$ are more correlated with $u_{2}$ (see the main components of $\mathbf{q}_{2}$ in Eq. (32)); and c) at $k=51, c I_{M X}\left(x_{2}\right)$ and $c I_{M X}\left(x_{6}\right)$ diagnose an excessive change in the normal operation point (though still following the correlation structure), because the contributions of $x_{2}$ and $x_{6}$ are more correlated with $t_{2}$ (see the main components of $\mathbf{p}_{2}$ in Eq. (32)).

\subsection{Application to a chemical process with feedback control}

The chemical process described by Yoon and MacGregor [31] is here adopted to evaluate the proposed method of process monitoring with fault detection and isolation. The process consists of a first-order chemical reaction carried out in a non-isothermal continuously-stirred tank reactor, where both the solute (A) and the solvent (S) are continuously fed into the reactor (Fig. 4). This example has widely been used in the literature to test other methods of fault detection and diagnosis; e.g. methods based on both linear [31] and nonlinear [14,32,33] LV models. The mathematical model of the process is given by [31]:

$$
\begin{aligned}
F= & F_{A}+F_{S}, \\
C_{A 0}= & \left(C_{A A} F_{A}+C_{A S} F_{S}\right) / F, \\
\frac{d C_{A}}{d t}= & \frac{F}{V} C_{A 0}-\frac{F}{V} C_{A}-k_{0} e^{-E / R T} C_{A}, \\
\frac{d T}{d t}= & \frac{F}{V}\left(T_{0}-T\right)-\frac{a F_{C}^{3 / 2}}{\left(F_{C}+a F_{C}^{1 / 2} / 2 \rho_{C} C_{P C}\right) V \rho C_{P}}\left(T-T_{C}\right) \\
& +\frac{\left(-\Delta H_{r}\right)}{\rho C_{P}} k_{0} e^{-E / R T} C_{A}, \\
Q= & \left(-\Delta H_{r}\right) V k_{0} e^{-E / R T} C_{A},
\end{aligned}
$$

with the following parameters: $V=1\left(\mathrm{~m}^{3}\right), \rho=10^{6}\left(\mathrm{~g} / \mathrm{m}^{3}\right), \rho_{C}=$ $10^{6}\left(\mathrm{~g} / \mathrm{m}^{3}\right), E / R=8330.1(\mathrm{~K}), C_{P}=1(\mathrm{cal} / \mathrm{gK}), C_{P C}=1(\mathrm{cal} / \mathrm{gK})$, $k_{0}=10^{10}\left(\mathrm{~m}^{3} / \mathrm{kmol} \times \min \right), a=1.678 \times 10^{6}(\mathrm{cal} / \mathrm{min} \times \mathrm{K})$, and $\Delta H_{r}=-1.3 \times 10^{7}(\mathrm{cal} / \mathrm{kmol})$.

The process is monitored from the following measurements: the inlet A flow rate $\left(F_{A}\right)$, the inlet $A$ concentration $\left(C_{A A}\right)$, the inlet solvent flow rate $\left(F_{S}\right)$, the $A$ concentration in the solvent $\left(C_{A S}\right)$, the reactant mixture temperature $\left(T_{0}\right)$, the cooling water temperature $\left(T_{C}\right)$, the coolant flow

\begin{tabular}{|c|c|c|c|c|c|c|}
\hline $\begin{array}{l}\text { Sample } \\
\text { point }\end{array}$ & $c I_{M X}()$. & $c I_{R X}()$. & $c I_{R Y 1}()$. & $c I_{R Y 2}()$. & $\begin{array}{l}\text { Diagnosis of the } \\
\text { abnormal event }\end{array}$ & $\begin{array}{l}\text { Diagnosed } \\
\text { class }\end{array}$ \\
\hline$k=11$ & - & $x_{1}, x_{6}$ & - & - & Faults in sensors of $x_{1}$ and $x_{6}$ & 1 \\
\hline$k=19$ & - & - & - & $y_{1}$ & Fault in the sensor of $y_{1}$ & 2 \\
\hline$k=27$ & - & $x_{2}$ & $y_{5}, y_{1}, x_{2}$ & - & Correlation changes in $\mathbf{x}^{\mathrm{a}}$ & 3 \\
\hline$k=35$ & - & - & $y_{2}, y_{4}$ & - & Change in the intrinsic gain & 4 \\
\hline$k=43$ & - & - & $x_{7}, x_{3}$ & $y_{1}$ & Correlation changes in $\mathbf{y}^{\mathrm{b}}$ & 5 \\
\hline$k=51$ & $x_{2}, x_{6}$ & - & - & - & Excessive operation change & 6 \\
\hline
\end{tabular}
rate $\left(F_{C}\right)$, the outlet concentration $\left(C_{A}\right)$, the outlet temperature $(T)$, and the reaction heat rate $(Q)$. These measurements are arranged as follows: $\mathbf{x}=\left[F_{A} C_{A A} F_{S} C_{A S} T_{0} T_{C} F_{C}\right]^{\prime}$ (process inputs), and $\mathbf{y}=\left[C_{A} T Q\right]^{\prime}$ (process

Table 4

Anomaly classification and identification of disturbed variables.

a Main changes occur in $x_{2}$

b Main changes occur in $y_{1}$.

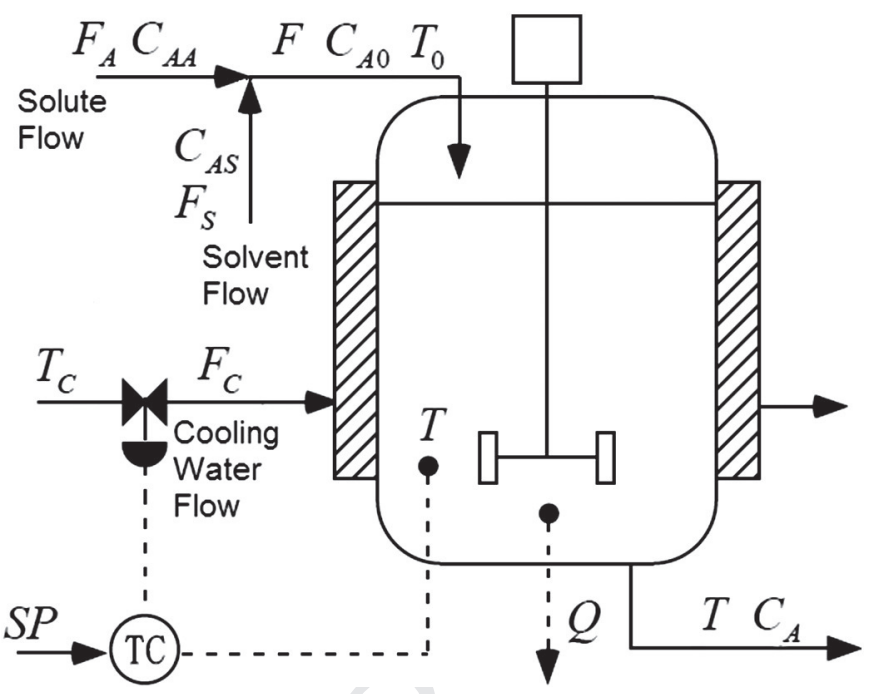

Fig. 4. Simulation example based on a dynamic process. Flow diagram of the process with 7 input- and 3 output measured variables.

outputs). Additionally, the following measured process disturbances 561 were selected: $C_{A A}, F_{S}, C_{A S}, T_{0}$, and $T_{C}$, which were simulated (under 562 both normal and fault conditions) through a first-order autoregressive 563 (AR) model, i.e.: $x_{i}(k)=\phi_{i} x_{i}(k-1)+\sigma_{e, i} e(k)$, where $e(k) \sim N(0,1) 564$ and $\sigma_{e, i}^{2}$ is the process variance, and $\phi_{i}$ is the AR parameter. Random 565 measurement noises are added to all process variables according 566 to: $x_{i, \text { meas }}(k)=x_{i}(k)+\sigma_{m, i} m(k)$ and $y_{i, \text { meas }}=y_{i}(k)+\sigma_{m, i} m(k), 567$ where $m(k) \sim N(0,1)$ and $\sigma_{m, i}^{2}$ is the noise variance. The parameters 568 of the disturbances and measurement noises are shown in Table 5. 569

The reaction temperature is regulated through a PI controller, 570 implemented as: $d F_{C} / d t=K_{C} d\left(S P_{T}-T\right) / d t+K_{C}\left(S P_{T}-T\right) / T_{l}$, with the 571 following parameters: $K_{C}=-1.5$ and $T_{I}=5.0$. The adopted initial 572 conditions are: $T_{0}=370.0 \mathrm{~K}$, and $C_{A}=0.8 \mathrm{kmol} / \mathrm{m}^{3}$. The remaining 573 variables are kept constant at the following values: $T_{C}=365.0 \mathrm{~K}, 574$ $F_{C}=15 \mathrm{~m}^{3} / \mathrm{min}, T=368.25 \mathrm{~K}, F_{S}=0.9 \mathrm{~m}^{3} / \mathrm{min}, F_{A}=0.1 \mathrm{~m}^{3} / \mathrm{min}, 575$ $C_{A}=0.8 \mathrm{kmole} / \mathrm{m}^{3}, C_{A S}=0.1 \mathrm{kmol} / \mathrm{m}^{3}$, and $C_{A A}=19.1 \mathrm{kmol} / \mathrm{m}^{3}$. All 576 variables were sampled at regular intervals of one minute. Under nor- 577 mal conditions, 200 samples were used to define the model calibration 578 dataset. The following PLSR model was identified in this work:

$\mathbf{y}(k)=\mathbf{Q B R}^{\prime} \mathbf{x}(k-1)$,

where $\mathbf{y}(k)$ represents the response vector at the discrete time $k$, and 580 $\mathbf{x}(k-1)$ is the predictor vector at $k$-1. Eq. (34) represents a static model 582 with a one-point delayed input. Based on criteria given by Godoy et al. 583 [18], three LVs were required to fit the PLSR model. Since the number 584 of LVs equals the number of process outputs, then the response space 585

Table 5

Parameters for simulating process disturbances and measurement noises.

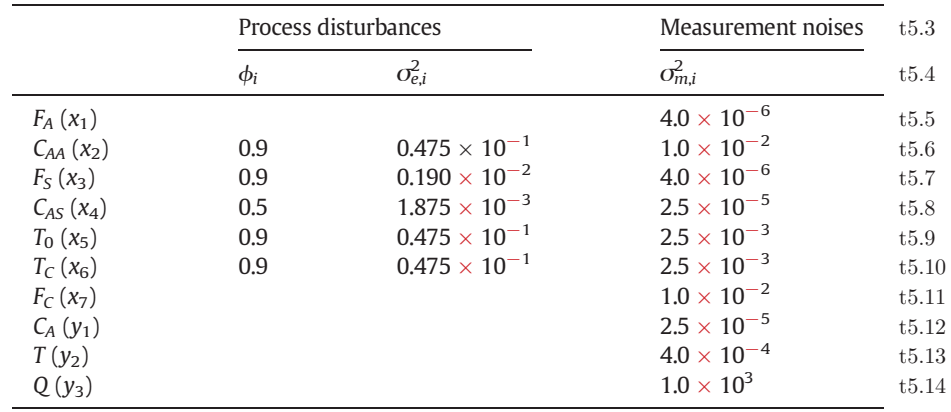


cannot be separated into two subspaces. Anyway, the monitoring technique can still be applied.

To evaluate the performance of the proposed technique, four types of faults are simulated as follows: F.1) a (non-propagating) sensor fault, simulated as a bias of $+2.4 \mathrm{~K}$ in $T_{0} ;$ F.2) a sensor fault (propagated by the PI control loop), simulated as a bias of $+1.5 \mathrm{~K}$ in $T$; F.3) a process change, simulated as a slow drift in the reaction kinetics, given by $k_{0}(k)=0.999 k_{0}(k-1)$; and F.4) a process disturbance, simulated as an unmeasured increment in the reactant mixture temperature, given by $T_{0, \text { in }}=T_{0}+0.03 \mathrm{k}$. According to the classification given by Yoon and MacGregor [31], F.1 is a simple fault while F.2, F.3, and F.4 are complex faults. The proposed cases are qualitatively similar to fault scenarios investigated in previous works [14,31-33]. Also, a quite similar process has been used to test a diagnosis technique based on a linear LV model, with the fault scenarios F.1 and F.2 [34].

For simplicity, each fault was introduced at $k=50$, and was kept until the end of the simulation. All cases were simulated along $400 \mathrm{~min}$, and therefore 400 samples were collected. The simulation results are depicted in Figs. 5-8. The first row of each figure represents the fault detection pattern, where the ordinates are normalized to $I_{T C} / I_{\alpha}$ and therefore the control limit is 1 . The three last rows of each figure constitute the basis of the diagnosis strategy based on the contribution analysis.

For the fault F.1), Fig. 5 shows a significant change in $c I_{R X}\left(x_{5}\right)$ for $k>50$, while both $c I_{M X}($.$) and c I_{R Y 1}($.$) remain close or slightly below 1$. The variable $x_{5}\left(=T_{0}\right)$ was identified as dominant, with a neat prevalence on $I_{R X}$ and smaller effects on the other subspaces.

In the case of F.2), Fig. 6 shows a rapid alarm in $I_{R Y 1}$ at $k=51$, while negligible contributions to $I_{M X}$ and $I_{R X}$ are observed. Around $30 \mathrm{~min}$ after the beginning of the fault $(k=51-80)$, the variable contributions to $I_{R Y 1}$ properly indicate the root source (i.e., the bias in $T$ ). Since $T$ is a controlled variable, the bias fault is gradually removed by effect of the closed-loop action, and the anomaly is then transferred to other variables. From $k=80$ onwards, the PI controller propagates the anomaly to the variable $F_{C}\left(x_{7}\right)$, which modified $T$ and $C_{A}$, as it is notified by $C I_{R Y 1}$. Additionally, an alert in $I_{M X}$ diagnoses a change in the operating point together with an alert in $I_{R X}$ that indicates a correlation change in $F_{C}\left(x_{7}\right)$ with respect to the remaining variables (i.e., $\left.x_{1} \ldots x_{6}\right)$. The disturbed $x_{7}$ is correctly diagnosed by the contributions to $I_{M X}$ and $I_{R X}$. The variable contributions to each component statistic exhibit transient re- 624 sponses until the process is settled to the new steady state. 625

The simulated fault F.3) corresponds to a gradual degradation of the 626 reaction rate, as typically caused by catalyst poisoning. The process 627 change was detected at $k \approx 170$, after collection of around 120 samples. 628 The high levels of $c I_{R Y 1}\left(y_{1}\right)$ and $c I_{R Y 1}\left(y_{3}\right)$ in Fig. 7 indicate that both $C_{A} 629$ and $Q$ cannot be adequately predicted. Since no predictor variable is at- 630 tributed to such changes, then it can be inferred that the anomaly is due 631 to the modification of some parameter of the plant ( $k_{0}$ in this case). 632 Note that $k_{0}$ is related to $T, C_{A}$, and $Q$ (see Eq. (33)), but $T$ is regulated to 633 $S P_{T}$ by the PI controller.

Fault F.4) consists in introducing a slow drift of $+0.03 \mathrm{~K} / \mathrm{min}$ in $T_{0} .635$ This continuous temperature rise indirectly causes an increase in the 636 inlet coolant flow rate $F_{C}\left(x_{7}\right)$, as required to keep the reactor tempera- 637 ture constant at its desired value. According to Fig. 8, a correlation loss 638 between $F_{C}$ and the remaining input variables is first diagnosed by a 639 high $c I_{R X}\left(x_{7}\right)$; then, a change in the operating point of $F_{C}$ is diagnosed 640 by a high $C I_{M X}\left(x_{7}\right)$.

The four analyzed example faults allow a direct comparison with 642 some detection and diagnosis studies previously-reported [14,31-34]. 643 Each of these works investigated a different (linear or nonlinear) LV 644 technique that was applied to the evaluation of only a limited number 645 of the described fault scenarios F.1 to F.4. More specifically, a Kernel 646 PCA technique was applied to F.1, F.2, and F.3 [14], a steady-state fault 647 signature technique was applied to F.1 and F.2 [31], a nonlinear 648 multiscale modeling technique was applied to F.1, F.3, and F.4 [33], and 649 a contribution plot without smearing effect was applied to F.1 and F.2 650 [34]. Besides, a technique based on Gaussian process LV models was eval- 651 uated onto the fault scenario F.2, but with purposes of fault detection 652 only [32]. In contrast, the technique presented in this work is linear 653 and successfully detects and diagnoses the four simulated fault scenarios. 654 In general, it was observed that the detection sensitivity (i.e., the minimal 655 disturbance that triggers an out-of-control of the index $I_{T C}$ ) is equivalent 656 to the sensitivity achieved by the individual approaches of [14,31-34] in 657 the cases of the fault scenarios F.1, F.2, and F.4. However, the present ap- 658 proach is more sensitive than that used in $[14,33]$ for the fault scenario 659 F.3, and allows adequate detection and diagnosis even under lower levels 660 of the reaction degradation. Additionally, it should be noticed that the 661

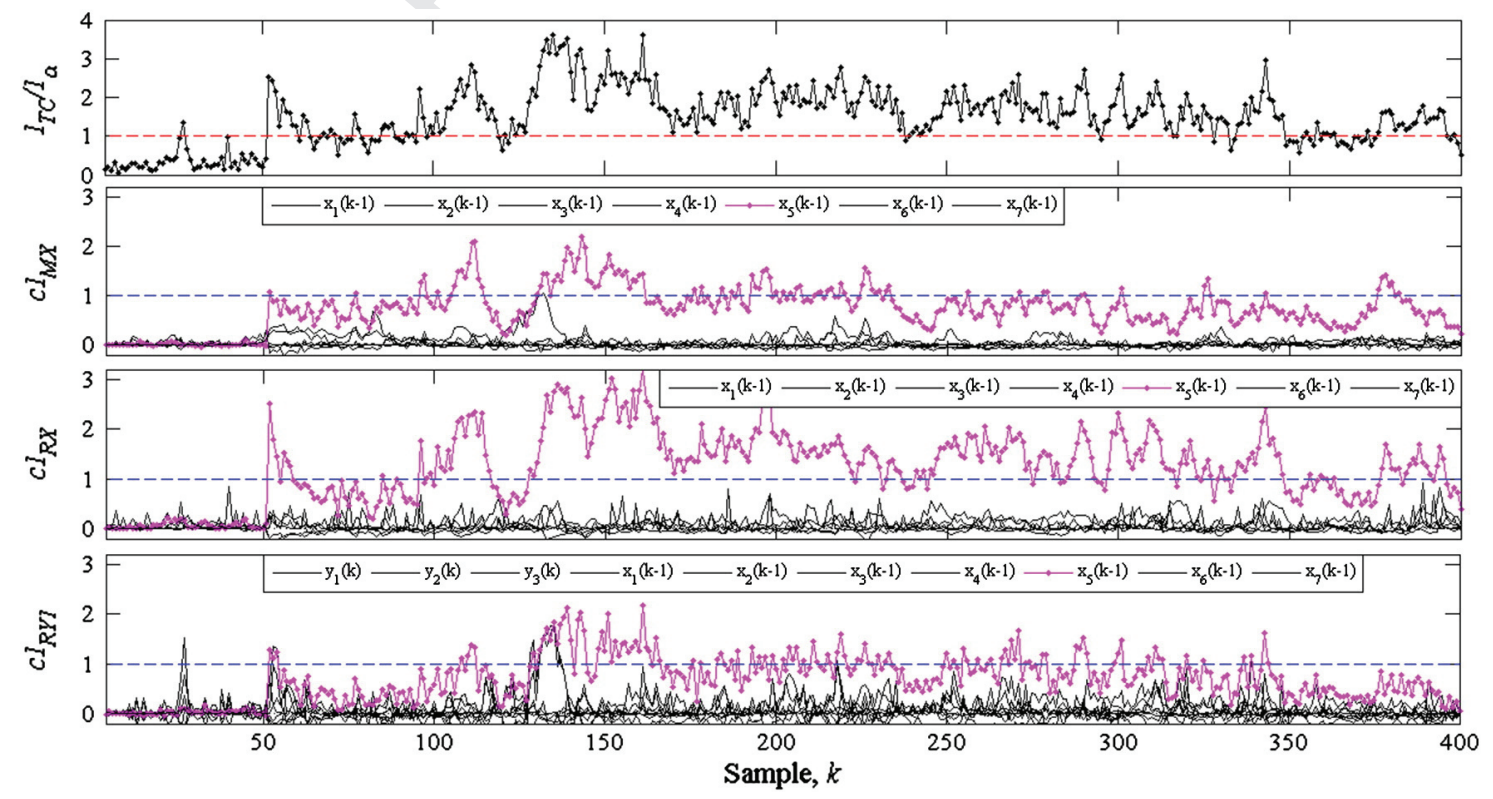

Fig. 5. Simulation of fault F.1): bias in the measurement of $T_{0}$. The contributions to the statistic $I_{R X}$ identify the variable $x_{5}\left(=T_{0}\right)$ as the source of the fault. 


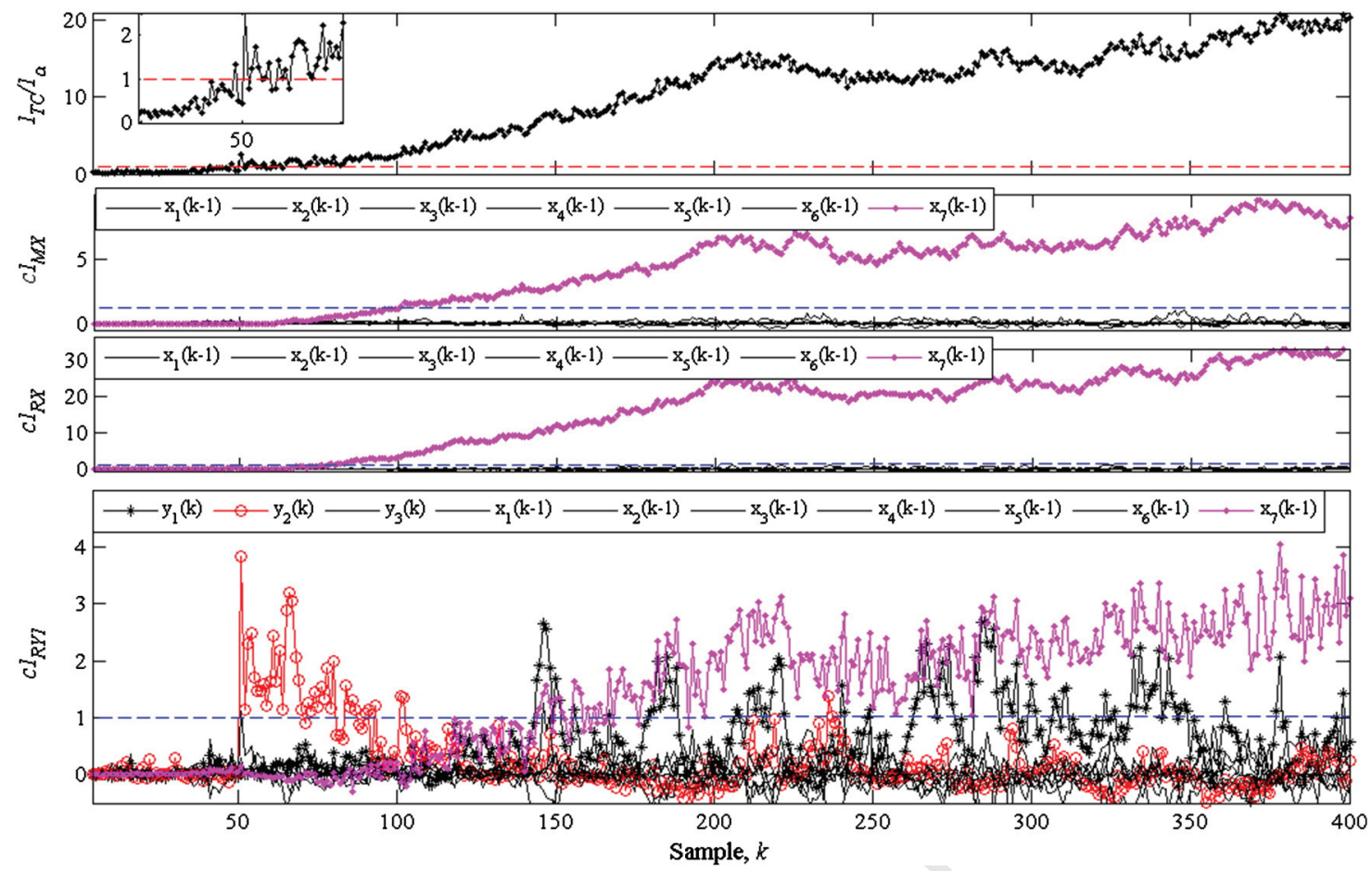

Fig. 6. Simulation of fault F.2): bias in the measurement of $T$. At $50<k<80$, the contributions to the statistic $I_{R Y 1}$ identify the variable $y_{2}(=T)$ as the source of the fault.

present detection and diagnosis procedure, implemented through an anomaly classification step followed by a further variable identification, is only based on theoretical considerations that enabled a proper diagnosis of several classes of faults (Table 4).

\section{Conclusions}

The proposed monitoring technique is based on a PLSR model initially designed under 'in-control' conditions, and is especially useful for monitoring processes that exhibit collinear measurements. The fundamentals of the method are based on detecting meaningful deviations of the measurements from their expected behaviors, which in turn serve for diagnosing abnormal events. The extension of the PLSR modeling strategy 672 consisted in a new PLS-decomposition that included more specific sta- 673 tistics, such as the distance to the regression model. By means of several 674 numerical simulations, it was verified that the proposed method was 675 effective for detecting and diagnosing different anomalies.

676

The proposed detection index, $I_{T C}$, involves a balanced merging of 677 several scaled metrics; and it represents a statistical distance that con- 678 siders the correlation structure of the process as well as three 679 Euclidean distances to the model. The analytical expression of $I_{T C} 680$ [Eq. (22)] contains an optimized combination of statistics. In fact, if a 681 new statistics were added (e.g., $T_{\widehat{u}}^{2}$ ), then the new combined index 682 would be: $I_{T C}^{*}=2 I_{M X}+I_{R X}+I_{R Y 1}^{u}+I_{R Y 2}=I_{T C}+I_{M X}$. Thus, the 683
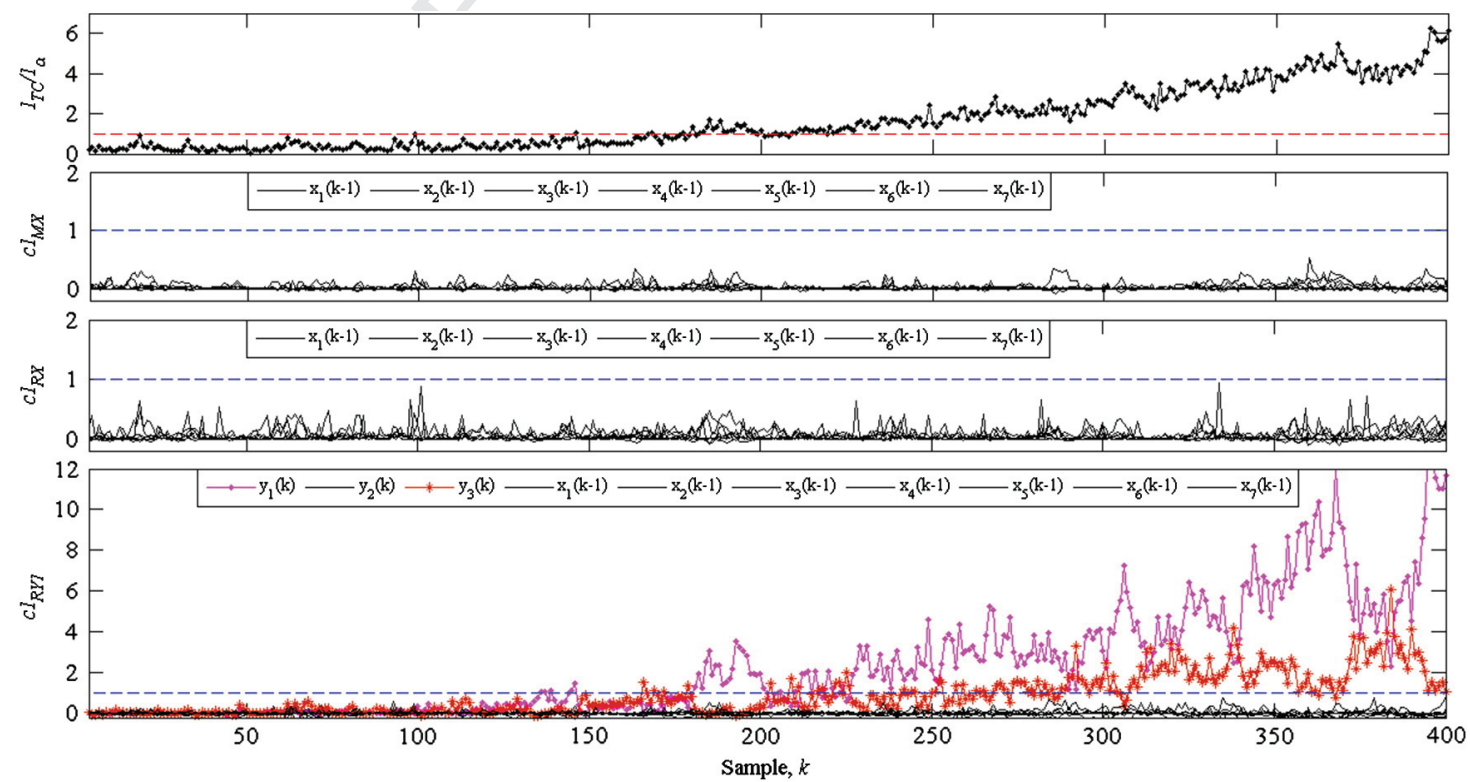

Fig. 7. Simulation of fault F.3): degradation of the reaction rate. At $k>170$, the contributions to the statistic $I_{R Y 1}$ indicate that predictions of the variables $y_{1}\left(=C_{A}\right)$ and $y_{3}(=Q)$ could be highly erroneous. 


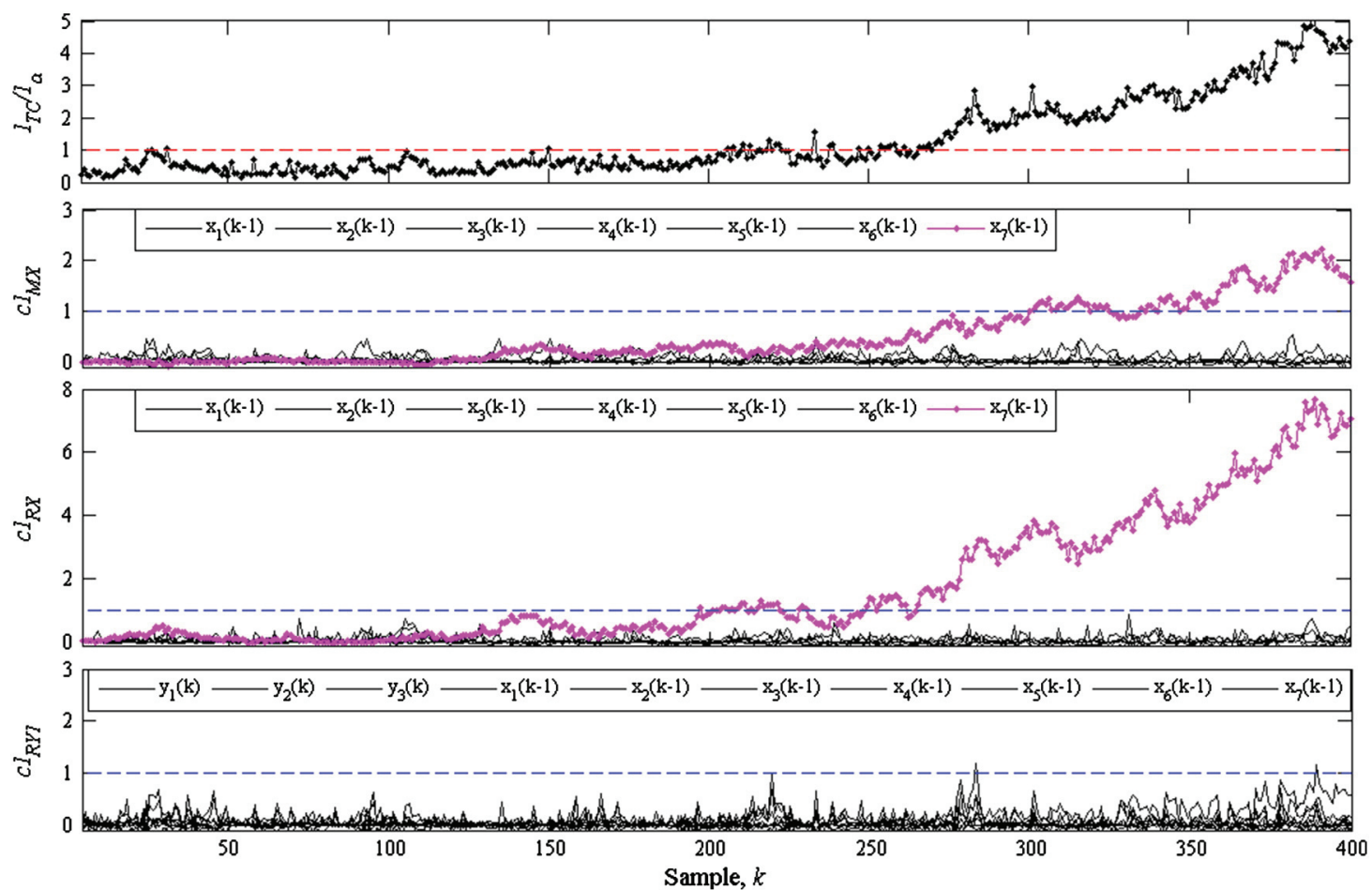

Fig. 8. Simulation of fault F.4): disturbance in $T_{0}$. At $k>200$, the contributions to the statistic $I_{R X}$ identify a correlation loss of the variable $x_{7}\left(=F_{C}\right.$ ) followed by a change in its operating point (as identified in $I_{M X}$ from $k=300$ onwards).

magnitude of $I_{M X}$ would be overweighed, and therefore the information contained in the remaining three statistics will be partially hidden.

In the application examples, only small deviations with respect to the nominal values were adopted, in order to evaluate the ability of the control volume associated to $I_{T C}$ for detecting the simulated errors. When an anomaly was present in the process, the combination of the alerts observed in the isolated statistics conforming $I_{T C}$ was efficiently used for classifying the perturbation source. Such preliminary diagnostic was then completed through the analysis of contributions to the alarmed statistics. Isolation of the disturbed variables was feasible through a decomposition of each component statistic in their contributions. The results obtained with two simulation examples show that the proposed technique is effective and accurate enough to be exploited with more emphasis in the future.

In summary, the proposed technique exhibits the following advantages: 1) it is an effective tool to simultaneously cope with quality monitoring, detection, and diagnosis of process faults, and isolation of multiple sensor faults; 2) it is based on a linear LV model and its calibration is relatively simple because only requires data taken from the process operating under normal conditions; and 3 ) the detection and diagnosis method is mainly based on the theoretical considerations exposed in the first part of this work and compiled in Table 2; instead of being based on recorded patterns of historical faults, such as in the fault signature methods. A limitation of the proposed technique is that it might not be advisable when the data collected under normal operating conditions are multimodal and/or when strong nonlinear correlations are present. However, it could be extended to dynamic models (e.g., by using dynamic PLS), and also to nonlinear models (e.g., by using kernel PLS).

\section{Acknowledgments}

The authors are grateful for the financial support received from CONICET, MinCyT, Universidad Nacional del Litoral, and Universidad Tecnológica Nacional (Argentina).

\section{Appendix A. Proofs of Lemma 1 and Theorems 1 and 2}

Proof 1. The oblique projector onto $\operatorname{Span}\{\mathbf{A}\}$ along $\operatorname{Span}\{\mathbf{B}\}$ can be 719 obtained through the following equation [27]:

$\Pi_{\mathbf{A} \mid \mathbf{B}}=\mathbf{A}\left(\mathbf{A}^{\prime} \Pi_{\mathbf{B}}^{\perp} \mathbf{A}\right)^{-1} \mathbf{A}^{\prime} \Pi_{\mathbf{B}}^{\perp}$

where $\Pi_{\mathbf{B}}^{\perp}$ is the orthogonal projector onto $\operatorname{Span}\{\mathbf{B}\}^{\perp}$.

Since $\mathbf{R}$ and $\mathbf{S}$ are full column rank, then:

$\Pi_{\mathbf{R}^{\perp}}^{\perp}=\Pi_{\mathbf{R}}=\mathbf{R}\left(\mathbf{R}^{\prime} \mathbf{R}\right)^{-1} \mathbf{R}^{\prime}$,

$\Pi_{\mathbf{S}^{\perp}}^{\perp}=\Pi_{\mathbf{S}}=\mathbf{S}\left(\mathbf{S}^{\prime} \mathbf{S}\right)^{-1} \mathbf{S}^{\prime}$

Since $\mathbf{P}^{\prime} \mathbf{R}=\mathbf{R}^{\prime} \mathbf{P}=\mathbf{I}$ (or $\left.\mathbf{Q}^{\prime} \mathbf{S}=\mathbf{S}^{\prime} \mathbf{Q}=\mathbf{I}\right)$, then substituting Eq. (A.2) 728 [or Eq. (A.3)] into Eq. (A.1) yield: $\Pi_{\mathbf{P} \mid \mathbf{R}^{\perp}}=\mathbf{P R}^{\prime}\left(\right.$ or $\left.\Pi_{\mathbf{Q} \mid \mathbf{S}^{\perp}}=\mathbf{Q} \mathbf{S}^{\prime}\right)$. Similarly, 729 we have $\Pi_{\mathbf{R}^{\perp} \mid \mathbf{P}}=\mathbf{I}-\mathbf{P R}^{\prime}$ ( or $\Pi_{\mathbf{S}^{\perp} \mathbf{Q}}=\mathbf{I}-\mathbf{Q S}^{\prime}$ ). $\square$

Partially, Lemma 1 has also been proved by Gang et al. [25].

Proof 2. Equations $(10,11)$ can be proved by taking into account 732 that: (i) $\operatorname{Span}\left\{\mathbf{I}-\mathbf{P R}^{\prime}\right\}=\operatorname{Span}\{\mathbf{R}\}^{\perp}$ and $\operatorname{Span}\left\{\mathbf{I}-\mathbf{Q S}^{\prime}\right\}=\operatorname{Span}\{\mathbf{S}\}^{\perp} 733$ (see Lemma 1); and (ii) the projections belong to complementary 734 subspaces, because $\operatorname{rank}\left(\mathbf{P R}^{\prime}\left(\mathbf{I}-\mathbf{P R}^{\prime}\right)\right)=\operatorname{dim}\left(S_{M X}\right)+\operatorname{dim}\left(S_{R X}\right)=m 735$ and $\operatorname{rank}\left(\mathbf{Q S}^{\prime}\left(\mathbf{I}-\mathbf{Q S}^{\prime}\right)\right)=\operatorname{dim}\left(S_{M Y}\right)+\operatorname{dim}\left(S_{R Y}\right)=$ p. Eq. (12) is direct- 736 ly derived from Eq. (7). $\square$

Proof 3. Substituting $\widehat{\mathbf{y}}=\mathbf{Q} \widehat{\mathbf{u}}$ into Eq. (21b), one obtains $D_{\widehat{\mathbf{y}}}=\widehat{\mathbf{u}}^{\prime} \mathbf{Q}^{\prime} 738$ $\left(\mathbf{Q} \Delta \mathbf{Q}^{\prime}\right)^{-} \mathbf{Q} \widehat{\mathbf{u}}$. The singular value decomposition of the (full-column 739 rank) matrix $\mathbf{Q}(p \times A)$ is $\mathbf{Q}^{\prime}=\mathbf{V}[\mathbf{\Sigma} \mathbf{0}] \mathbf{W}^{\prime}$, where $\mathbf{\Sigma}(A \times A)$ is a non- 740 singular diagonal matrix, and $\mathbf{W}(p \times p)$ and $\mathbf{V}(A \times A)$ are orthonormal 741 matrices. Then,

$$
\begin{aligned}
\mathbf{Q}^{\prime}\left(\mathbf{Q} \Delta \mathbf{Q}^{\prime}\right)^{-} \mathbf{Q} & =\mathbf{V}\left[\begin{array}{ll}
\mathbf{\Sigma} & \mathbf{0}
\end{array}\right] \mathbf{W}^{\prime}\left(\mathbf{W}\left[\begin{array}{c}
\mathbf{\Sigma} \\
\mathbf{0}
\end{array}\right] \mathbf{V}^{\prime} \Delta \mathbf{V}\left[\begin{array}{ll}
\mathbf{\Sigma} & \mathbf{0}
\end{array}\right] \mathbf{W}^{\prime}\right)^{-} \mathbf{W}\left[\begin{array}{c}
\mathbf{\Sigma} \\
\mathbf{0}
\end{array}\right] \mathbf{V}^{\prime}, \\
& =\mathbf{V} \mathbf{\Sigma}\left(\mathbf{\Sigma} \mathbf{V}^{\prime} \Delta \mathbf{V} \mathbf{\Sigma}\right)^{-1} \mathbf{\Sigma} \mathbf{V}^{\prime}=\Delta^{-1} .
\end{aligned}
$$


Therefore, $D_{\widehat{\mathbf{y}}}=\widehat{\mathbf{u}}^{\prime} \Delta^{-1} \widehat{\mathbf{u}}=T_{\widehat{\mathbf{u}}}^{2}$. By combining Eqs. (18) and (15b) with $\widehat{\mathbf{u}}=\mathbf{B t}$ S, we obtain $T_{\widehat{\mathbf{u}}}^{2}=\mathbf{t}^{\prime} \mathbf{B}\left(\mathbf{B}^{-1} \boldsymbol{\Lambda}^{-1} \mathbf{B}^{-1}\right) \mathbf{B t}=T_{\mathbf{t}}^{2}$. By replacing $\widehat{\mathbf{x}}=$ Pt into Eq. (21a): $D_{\widehat{\mathbf{x}}}=\mathbf{t}^{\prime} \mathbf{P}^{\prime}\left(\mathbf{P} \mathbf{\Lambda} \mathbf{P}^{\prime}\right)^{-} \mathbf{P t}$. Similarly to the derivation of Eq. (A.4), it is obtained: $\mathbf{P}^{\prime}\left(\mathbf{P} \boldsymbol{\Lambda} \mathbf{P}^{\prime}\right)^{-} \mathbf{P}=\boldsymbol{\Lambda}^{-1}$; hence $D_{\widehat{\mathbf{x}}}=\mathbf{t}^{\prime} \boldsymbol{\Lambda}^{-1} \mathbf{t}=T_{\mathbf{t}}^{2}$. From all these equalities, Theorem 2 is proven. $\square$

\section{Appendix B. Control limits}

To calculate the confidence limits we assume that $\mathbf{x}$ and $\mathbf{y}$ approximately follow multivariate normal behaviors. The $T^{2}$ control limit at the $\alpha$ significance level is given by [5] $\tau_{\alpha}^{2}=$ $\left[\left(A N^{2}-A\right) /\left(N^{2}+N A\right)\right] F_{A, N-A}(\alpha)$, where $F_{A, N-A}($.$) is the F-distri-$ bution with $A$ and $N-A$ degrees of freedom. On the other hand, the $\alpha$-control limit of $S P E_{\mathbf{x}}$ is given by [7] $\delta_{\mathbf{x}, \alpha}^{2}=\left(\sigma^{2} / 2 \nu\right) \chi_{2 \nu^{2} / \sigma^{2}}^{2}$ $(\alpha)$ where $\nu$ and $\sigma^{2}$ are the sample average and variance estimated with $N$ observations of $S P E_{\mathbf{x}}$, and $\chi_{2 \nu^{2} / \sigma}^{2}($.$) is the \chi^{2}$-distribution with $2 \nu^{2}$ / $\sigma^{2}$ degrees of freedom. Similarly, the control limits of $S P E_{\mathbf{y} 1}$ and $S P E_{\mathbf{y} 2}$ are calculated.

The $100(1-\alpha) \%$ control limit of the $I_{T C}$ is given by [11]

$I_{\alpha}=g \chi_{h}^{2}(\alpha)$

with $g$ and $h$ given by:

$g=\frac{\operatorname{tr}\left[\left(\mathbf{R}_{\left[\mathbf{y}^{\prime} \mathbf{x}^{\prime}\right]} \boldsymbol{\Phi}\right)^{2}\right]}{\operatorname{tr}\left(\mathbf{R}_{\left[\mathbf{y}^{\prime} \mathbf{x}^{\prime}\right]} \boldsymbol{\Phi}\right)}, h=\frac{\left[\operatorname{tr}\left(\mathbf{R}_{\left[\mathbf{y}^{\prime} \mathbf{x}^{\prime}\right]} \boldsymbol{\Phi}\right)\right]^{2}}{\operatorname{tr}\left[\left(\mathbf{R}_{\left[\mathbf{y}^{\prime} \mathbf{x}^{\prime}\right]} \boldsymbol{\Phi}\right)^{2}\right]}$

where $\mathbf{R}_{\left[\mathbf{y}^{\prime} \mathbf{x}^{\prime}\right]}$ is the correlation matrix of the extended vector $\left[\mathbf{y}^{\prime} \mathbf{x}^{\prime}\right]^{\prime}$ and $\Phi$ is equal to

$\boldsymbol{\Phi}=\left[\begin{array}{cc}\mathbf{S} \mathbf{Q}^{\prime} \mathbf{Q} \mathbf{S}^{\prime} / \delta_{\mathbf{y} 1, \alpha}^{2}+\left(\mathbf{I}-\mathbf{Q S}^{\prime}\right)^{\prime}\left(\mathbf{I}-\mathbf{Q} \mathbf{S}^{\prime}\right) / \delta_{\mathbf{y} 2, \alpha}^{2} & -\mathbf{S Q}^{\prime} \mathbf{Q B B} \mathbf{R}^{\prime} / \delta_{\mathbf{y} 1, \alpha}^{2} \\ -\mathbf{R B Q} \mathbf{Q}^{\prime} \mathbf{Q} \mathbf{S}^{\prime} / \delta_{\mathbf{y} 1, \alpha}^{2} & \boldsymbol{\Phi}_{22}\end{array}\right]$
$\Phi_{22}=\mathbf{R} \boldsymbol{\Lambda}^{-1} \mathbf{R}^{\prime} / \tau_{\alpha}^{2}+\left(\mathbf{I}-\mathbf{P R}^{\prime}\right)^{\prime}\left(\mathbf{I}-\mathbf{P} \mathbf{R}^{\prime}\right) / \delta_{\mathbf{x}, \alpha}^{2}+\mathbf{R B Q}^{\prime} \mathbf{Q} \mathbf{B} \mathbf{R}^{\prime} / \delta_{\mathbf{y} 1, \alpha}^{2}$

In all cases, the control limits were set at the 99 percentile $(\alpha=0.01)$.

\section{References}

[1] S.J. Qin, Survey on data-driven industrial process monitoring and diagnosis, Annual Reviews in Control 36 (2012) 220-234.

[2] J. Kresta, J.F. MacGregor, T.E. Marlin, Multivariate statistical monitoring of process operating performance, Canadian Journal of Chemical Engineering 69 (1991) 35-39.

[3] P. Nomikos, J.F. MacGregor, Monitoring batch process using multiway principal component analysis, AIChE Journal 40 (1994) 1361-1375.

[4] P. Nomikos, J.F. MacGregor, Multivariate SPC charts for monitoring batch processes, Technometrics 37 (1995) 41-59.

[5] T. Kourti, J.F. MacGregor, Process analysis, monitoring and diagnosis using multivariate projection methods, Chemometrics and Intelligent Laborary Systems 28 (1995) 3-21.
[6] T. Kourti, P. Nomikos, J.F. MacGregor, Analysis, monitoring and fault diagnosis 781 of batch process using multiblock and multiway PLS, Journal of Process Control 5782 (1995) 277-284.

[7] T. Kourti, Application of latent variable methods to process control and multivariate 784 statistical process control in industry, International Journal of Adaptive Control and 785 Signal Processing 19 (2005) 213-246. 786

[8] V. Venkatasubramanian, R. Rengaswamy, S.N. Kavuri, K. Yin, A review of process 787 fault detection and diagnosis Part III: process history based methods, Computers 788 and Chemical Engineering 27 (2003) 327-346.

[9] A.L. Pomerantsev, O.Y. Rodionova, Process analytical technology: a critical view of 790 the chemometricians, Journal of Chemometrics 26 (2012) 299-310.

[10] C.F. Alcala, S.J. Qin, Reconstruction-based contribution for monitoring, Automatica 792 45 (2009) 1593-1600

[11] C.F. Alcala, S.J. Qin, Analysis and generalization of fault diagnosis methods for pro- 794 cess monitoring, Journal of Process Control 21 (2011) 322-330. 795

[12] H.H. Yue, S.J. Qin, Reconstruction-based fault identification using a combined index, 796 Industrial and Engineering Chemistry Research 40 (2001) 4403-4414. 797

[13] J.M. Lee, C.K. Yoo, I.B. Lee, Statistical process monitoring with independent compo- 798 nent analysis, Journal of Process Control 14 (2004) 467-485. 799

[14] C.F. Alcala, S.J. Qin, Reconstruction-based contribution for process monitoring with 800 kernel principal component analysis, Industrial and Engineering Chemistry Re- 801 search 49 (2010) 7849-7857.

15] L. Wang, H. Shi, Multivariate statistical process monitoring using an improved inde- 803 pendent component analysis, Chemical Engineering Research and Design 88 (2010) 804 403-414.

[16] S. Wold, M. Sjöström, L. Eriksson, PLS-regression: a basic tool of chemometrics, 806 Chemometrics and Intelligent Laborary Systems 58 (2001) 109-130. 807

[17] H.A.L. Kiers, A.K. Smilde, A comparison of various methods for multivariate 808 regression with highly collinear variables, Statistical Methods and Applications 809 16 (2007) 193-228.

[18] J.L. Godoy, R.J. Minari, J.R. Vega, J.L. Marchetti, Multivariate statistical monitoring of an 811 industrial SBR Process. Soft-sensor for production and rubber quality, Chemometrics 812 and Intelligent Laborary Systems 107 (2011) 258-268.

[19] T. Mehmood, K.H. Liland, L. Snipen, S. Sæbø, A review of variable selection methods 814 in Partial Least Squares Regression, Chemometrics and Intelligent Laborary Systems 815 118 (2012) 62-69.

[20] J. Gabrielsson, N.-O. Lindberg, T. Lundstedt, Review: multivariate methods in 817 pharmaceutical applications, Journal of Chemometrics 16 (2002) 141-160. 818

[21] J.A. Lopes, P.F. Costa, T.P. Alves, J.C. Menezes, Chemometrics in bioprocess engineer- 819 ing: process analytical technology (PAT) applications, Chemometrics and Intelligent 820 Laborary Systems 74 (2004) 269-275.

[22] B. Junker, H. Wang, Bioprocess monitoring and computer control: key roots of the 822 current PAT initiative, Biotechnology and Bioengineering 95 (2006) 226-261. 823

[23] A. AlGazzawi, B. Lennox, Model predictive control monitoring using statistics, 824 Journal of Process Control 19 (2009) 314-327.

[24] H.W. Lee, M.W. Lee, J.M. Park, Multi-scale extension of PLS algorithm for advanced 826 on-line process monitoring, Chemometrics and Intelligent Laborary Systems 98827 (2009) 201-212.

[25] L.G. Gang, S.J. Qin, D. Zhou, Geometric properties of partial least squares for process 829 monitoring, Automatica 46 (2010) 204-210. 830

[26] P. Geladi, B. Kowalski, Partial least-squares regression: a tutorial, Analytica Chimica 831 Acta 185 (1986) 1-17.

[27] D. Meyer, Matrix Analysis and Applied Linear Algebra, SIAM, USA, 2000.

[28] I.C.F. Ipsen, C.D. Meyer, The angle between complementary subspaces, American 834 Mathematical Monthly 102 (1995) 904-911. 835

[29] J.A. Westerhuis, S.P. Gurden, A.K. Smilde, Generalized contribution plots in multivar- 836 iate statistical process monitoring, Chemometrics and Intelligent Laborary Systems 837 51 (2000) 95-114.

[30] C.R. Alvarez, A. Brandolin, M.C. Sánchez, On the variable contributions to the 839 D-statistic, Chemometrics and Intelligent Laborary Systems 88 (2007) 189-196. 840

[31] S. Yoon, J.F. MacGregor, Fault diagnosis with multivariate statistical models part I: 841 using steady state fault signatures, Journal of Process Control 11 (2001) 387-400. 842

[32] J. Serradilla, J.Q. Shi, A.J. Morris, Fault detection based on Gaussian process latent var- 843 iable models, Chemometrics and Intelligent Laborary Systems 109 (2011) 9-21. 844

[33] S.W. Choi, J. Morris, I. Lee, Nonlinear multiscale modelling for fault detection and 845 identification, Chemical Engineering Science 63 (2008) 2252-2266. 846

[34] J. Liu, Fault diagnosis using contribution plots without smearing effect on non-faulty 847 variables, Journal of Process Control 22 (2012) 1609-1623. 